\title{
Measuring capabilities in health and physical activity promotion: a systematic review
}

\author{
M. Till ${ }^{*}$, K. Abu-Omar, S. Ferschl, A. K. Reimers and P. Gelius
}

\begin{abstract}
Background: The capability approach by Amartya Sen and Martha Nussbaum has gained increasing attention in the field of public health. As it combines individual, social and structural factors and shifts the focus of attention from the actual behavior towards available options for health behaviors that people can actually choose from, it may help advance our understanding of complex health issues.
\end{abstract}

Objectives: The aim of this article is to identify and describe tools available to measure capabilities within the context of health, with a specific focus on capabilities for health-enhancing physical activity.

Method: We conducted a systematic literature review using 11 databases covering scientific journal articles published in English or German between the years 2000 and 2020 with a focus on capabilities for health or physical activity.

Results: We found a total of 51 articles meeting our inclusion criteria. Four articles measured capabilities using qualitative methods, one combined qualitative and quantitative methods, while the rest used quantitative methods. We identified a total 11 different capability questionnaires, all showing moderate to good validity/reliability. Only one questionnaire and one interview-based tool specifically dealt with capabilities for health enhancing physical activity.

Conclusion: Although we were able to identify measurement tools for capabilities in health, this review has shown that there is no generic tool available for the measurement across all population- and age-groups, and tools focusing on physical activity are scarce. However, our results can be used as guide for future projects that aim at measuring capabilities.

Keywords: Capability approach, Public health, Measurement, Operationalization, Physical activity, health outcomes, Questionnaires

\section{Background}

Over the last years, the capability approach - originally developed by Amartya Sen [1] in welfare economics has gained increasing attention in the field of health and has been used in multiple health promotion projects [2-

\footnotetext{
* Correspondence: maike.till@fau.de

Department of Sport Science and Sport, Friedrich-Alexander-University, Erlangen Nuremberg, Gebbertstraße 123b, 91058 Erlangen, Germany
}

6]. A recent review by Helter et al. [7] highlights this growing relevance of the capability approach in health promotion, particularly regarding its use within health economic evaluation of projects. The capability approach shifts the focus of attention from an individual's actual behavior - the realization of "various things a person may value being or doing" [8], e. g. having a healthy diet (called "achieved functionings") - towards the real opportunities - "various combinations of functionings that

(c) The Author(s). 2021 Open Access This article is licensed under a Creative Commons Attribution 4.0 International License, which permits use, sharing, adaptation, distribution and reproduction in any medium or format, as long as you give appropriate credit to the original author(s) and the source, provide a link to the Creative Commons licence, and indicate if changes were made. The images or other third party material in this article are included in the article's Creative Commons licence, unless indicated otherwise in a credit line to the material. If material is not included in the article's Creative Commons licence and your intended use is not permitted by statutory regulation or exceeds the permitted use, you will need to obtain permission directly from the copyright holder. To view a copy of this licence, visit http://creativecommons.org/licenses/by/4.0/ The Creative Commons Public Domain Dedication waiver (http://creativecommons.org/publicdomain/zero/1.0/) applies to the data made available in this article, unless otherwise stated in a credit line to the data. 
the person can achieve" [8] (called "capabilities") - available to individuals to choose from.

The shift of focus from people's behavior towards their real opportunities, that they can value and realize, can be particularly beneficial in the field of health promotion. In the context of this paper, we look at the capability approach from the perspective of physical activity (PA). PA is commonly defined as "any bodily movements produced by skeletal muscles that result in energy expenditure" [9] and has been generally proven to have a positive impact on people's health, e. g. in relation to obesity, non-communicable diseases (e. g. diabetes, highblood pressure), cardio-respiratory health, cancer, mental health and all-cause mortality [10, 11]. Healthenhancing PA (HEPA) may come in many forms and shapes across multiple domains, e. g. during leisure time (e. g. sports, walks or hiking), at the workplace, during transport (e. g. biking to school), or at home (e. g. gardening) [12].

Current efforts to promote PA, however, tend to focus on "downstream" interventions (e. g. physical education in school or structured PA classes for older people) that promise to have immediate effects on the target group's health [13]. However, such interventions focus mainly on outcome improvement, i. e. achieved health functionings, and tend to neglect the environmental or social components that led to the outcome in the first place. In doing so, such interventions may be less sustainable than more "upstream" interventions whose effects cannot immediately be measured in terms of target group behavior change (e. g. those that initiate infrastructure change [14] or that increase individuals' physical literacy, i. e. their "motivation, confidence, physical competence, knowledge and understanding to value and take responsibility for maintaining purposeful physical pursuits/activities throughout the lifecourse" [15]). To achieve sustainable behavior change, there is a need to extend the focus of HEPA interventions from focusing solely on outcomes (e. g. steps, hours spent being physically active, effects on weight etc.) towards also considering the capabilities of target groups to engage in desired behavior or to achieve valued states of being.

The capability approach may help achieve this shift of focus by pointing to the benefits in terms of capabilities for healthy behavior [2] or, as in our specific case, HEPA. It explicitly respects people's freedom to decide for or against a healthy behavior and looks at available or unavailable components which may have led to the specific outcome. Therefore, applying the capability approach within a health promotion project may enhance the target group's compliance by focusing on how to positively change the opportunities for health that they consider meaningful and desirable, rather than merely "forcing" them to behave in a healthy manner (e. g. through mandatory physical education in schools) to achieve positive change of health outcome.

In general, a person's capabilities for health enhancing behavior can be assumed to be based on a set of capitals or resources [5] that are "translated" into capabilities through three sets of conversion factors [6]: (1) individual (e. g. physical condition, biological health or health literacy), (2) social (e. g. norms and values, social practices or political rules), and (3) environmental factors (e. g. climate, pollution, infrastructure). However, operationalizing a concept as complex as the capability approach [3] (or, to give another example, Antonovsky's [16] "sense of coherence") for actual measurement is challenging, as it is rather theoretical in nature and underspecified (potentially by design) with respect to empirical application. Nonetheless, the increasing popularity of the capability approach in health and PA promotion obliges us to assess not only health status and indicators of behavior but also the available opportunities that people have to realize healthy behavior.

The aim of this paper is to support researchers in health and HEPA promotion who intend to use the capability approach by (1) systematically identifying all currently available tools to measure capabilities for health, well-being, and PA, (2) providing an overview of the main features of these tools as well as their psychometric properties, and applicability to different areas, and (3) discussing how the identified capability measures can be specifically used in the field of HEPA promotion by future researchers.

\section{Methods}

Research for this paper was conducted in the context of Capital4Health, a research consortium funded by the German Federal Ministry of Education and Research [01EL1421A-F] which aimed at promoting active lifestyles in four different settings across the life-course using the capability approach. A project (CAPCOM, [01EL1421A]) tasked with fostering cooperation in the consortium conducted the systematic review at hand in order to strengthen its common methodological base. The presented work followed the Preferred Reporting Items for Systematic-Reviews and Meta-Analyses (PRIS MA) guidelines [17].

An initial exploratory search for instruments to measure capabilities specifically for PA indicated that only a limited number of instruments were dedicated to this topic, we therefore decided to broaden the search to include capability measurement tools for health in general. This expansion may seem radical but was a logical next step given our health-centered perspective on PA and HEPA [12]. As options for measuring capabilities for PA are limited, gathering information on available measurement tools for the general capabilities of health and 
well-being will enable the identification of tools that can be adapted to PA or, in cases where adaptation is difficult, provide valuable lessons for the future development of new specific capability measurement tools for HEPA.

Supported by a university librarian, research team developed a set of search strings consisting of variations of the terms "capability approach", "measurement", "health" and "physical activity" combined with Boolean operators. A full version of the search term is provided in the appendix. On $14^{\text {th }}$ of October 2020, searches were conducted on the following databases: APA Psycinfo, Psychology and Behavioral Sciences Collection, SPORTDiscus, and APA PsycArticles via EBSCOhost, Applied Social Science Index \& Abstracts, Sociological Abstracts, Social Services Abstracts, Worldwide Political Science Abstracts, International Bibliography of the Social Science, and the Sports Medicine \& Education Index via ProQuest, and Pubmed.

Table 1 summarizes the inclusion/exclusion criteria applied to the results. Articles were included if they (a) were published between January 2000 and October 2020; (b) were written in English or German; (c) were scientific journal articles; (d) had a clear focus on the operationalization of the capability approach within the context of health or HEPA; and referred to any (e) population, (f) setting, or (g) country.

Two researchers independently screened all titles/abstracts based on the inclusion/exclusion criteria and discussed their results to resolve disagreement. Two researchers then independently screened the full texts of all remaining papers and discussed their results to reach consensus on the articles to be included for detailed analysis. In addition, the lead author carried out a supplementary hand search. Results of the latter were doublechecked by another researcher. The included final search results were imported into Endnote X9 and analyzed regarding (i) the proposed types of measurement instruments for capabilities, (ii) the development process employed to develop these instruments, and (iii) the empirically tested validity, reliability, and responsiveness of the instruments among different target groups.

For better comparison, in the context of this paper, we rated instrument quality as follows: construct validity was categorized as "good" when correlations with any chosen other instrument had shown to be at least moderate and significant, or when its chi-square analysis had shown to be significant at the $5 \%$ level [18]. We only rated the outcomes reported in the respective paper but not the measurement tool used for the comparison. Discriminant validity was rated as "good" when the instrument showed a significant (at least $p<.01$ ) distinction between different areas. Internal consistency with $\alpha>.7$ was considered "good" [19], as well as test-retest reliability with a moderate (>.41) Cohen's kappa [20] or an intraclass-correlation coefficient over .75 [21].

\section{Results}

The search yielded in a total of $N=11,354$ hits matching the search terms across all eleven databases. After removing all duplicates, a total of 8515 articles remained for screening. Researchers had substantial agreement on title/abstract screening (Cohen's $\mathrm{k}=0.66$ ), disagreeing mostly on the use of the capability approach within a paper [22]. This step yielded a total of 101 articles eligible for full-text screening. Researchers had moderate agreement in full-text screening (Cohen's $\mathrm{k}=0.44$ ) [20], leading to the exclusion of another 55 articles. Disagreement on inclusion or exclusion was mostly about the level of operationalization of the capability approach in papers, i. e. whether articles actually provided a fullfledged measurement tool or merely a theoretical framework. Five additional articles were identified during hand search, resulting in a total of $N=51$ articles included in this review, covering either the development of instruments for measuring capabilities according to the capability approach or psychometric properties. A visual representation of the search is shown in Fig. 1 using the PRISMA-flowchart [17].

Table 1 Inclusion and exclusion criteria

\begin{tabular}{|c|c|c|}
\hline Criterion & Inclusion & Exclusion \\
\hline Time & January 2000 - October 2020 & Studies before 2000 and after October 2020 \\
\hline Language & English, German & Any other language \\
\hline $\begin{array}{l}\text { Type of } \\
\text { publication }\end{array}$ & Journal Articles & Scientific papers published outside a journal \\
\hline Focus of study & $\begin{array}{l}\text { - Operationalization of the Capability Approach in terms of Amartya } \\
\text { Sen/ Martha Nussbaum } \\
\text { - Tool to measure Capabilities for health/heath enhancing physical } \\
\text { activity }\end{array}$ & $\begin{array}{l}\text { - Outside context of health } \\
\text { - Pure article on theory without operationalization of the } \\
\text { capability approach }\end{array}$ \\
\hline $\begin{array}{l}\text { Study } \\
\text { population }\end{array}$ & Any Population & Nil \\
\hline Setting & Any Setting & Nil \\
\hline Country & Any country & Nil \\
\hline
\end{tabular}



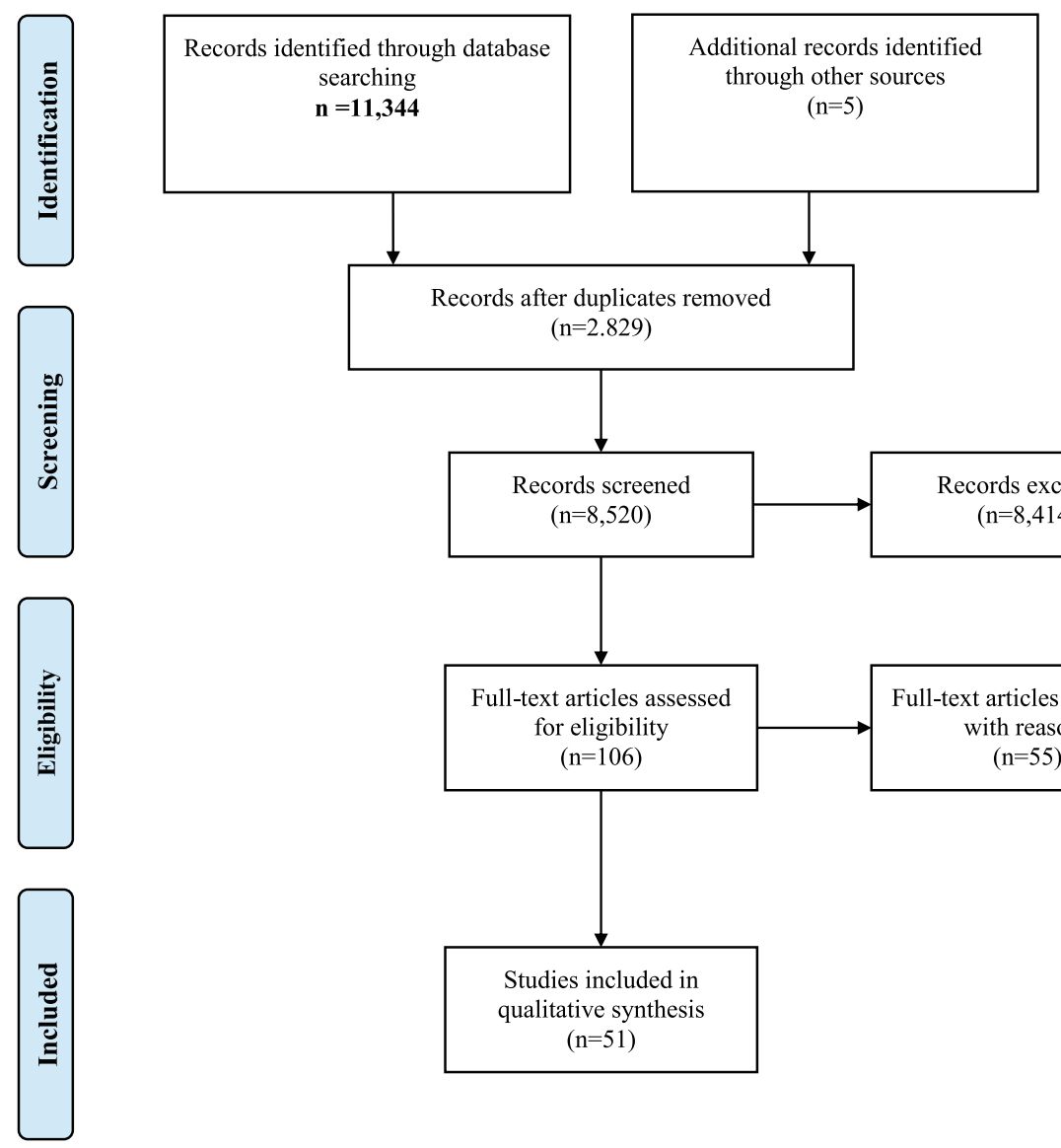

Records after duplicates removed $(n=2.829)$
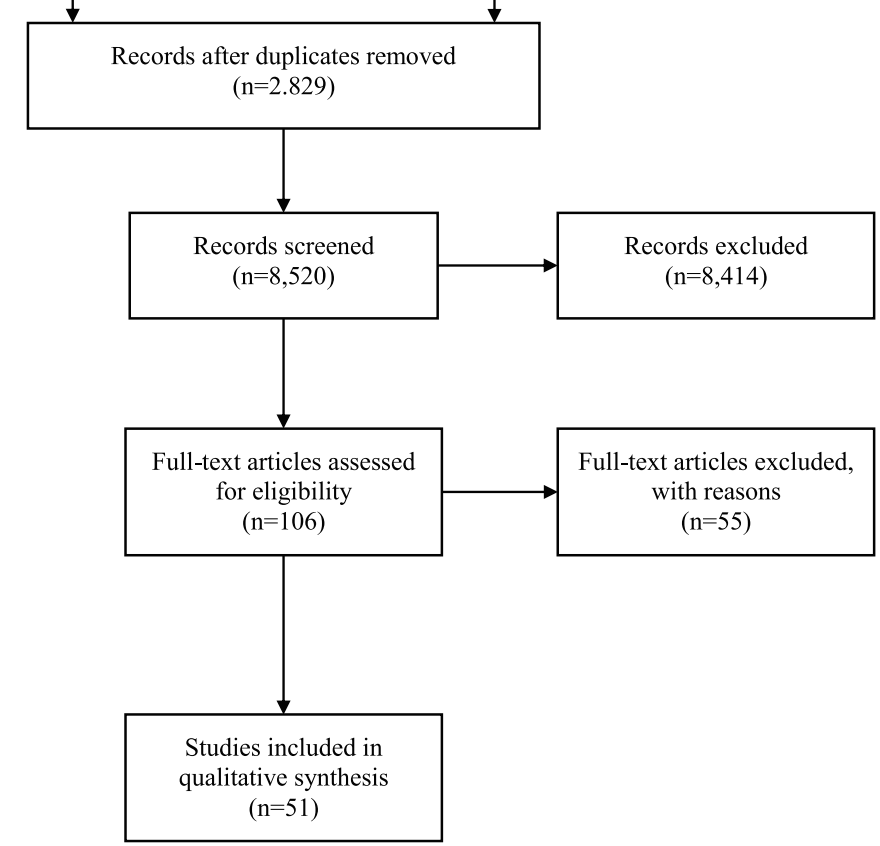

Fig. 1 Literature search flow chart based on PRISMA [13]

Table 2 Distribution of measurement tools

\begin{tabular}{|c|c|c|c|c|}
\hline \multirow{3}{*}{ Capability Measurement Tools } & \multirow[b]{2}{*}{ Qualitative tools } & \multicolumn{3}{|l|}{ No. } \\
\hline & & 1 & \multicolumn{2}{|c|}{ Interviews [23-26] } \\
\hline & & 2 & \multicolumn{2}{|c|}{ Videography [27] } \\
\hline & Mixed-Method & 3 & \multicolumn{2}{|c|}{ Questionnaire and Interviews [28] } \\
\hline & Quantitative tools & 4 & \multicolumn{2}{|c|}{ Questionnaire combinations (secondary data) $[21,28-30]$} \\
\hline & & 5 & \multirow[t]{4}{*}{ ICECAP } & ICECAP-O $[31]^{*}$ \\
\hline & & 6 & & ICECAP-A [32] $]^{*}$ \\
\hline & & 7 & & ICECAP-SCM $[33]^{*}$ \\
\hline & & 8 & & ICECAP-FC $[34]^{*}$ \\
\hline & & 9 & \multirow[t]{3}{*}{ OCAP } & OCAP $[35]^{*}$ \\
\hline & & 10 & & OCAP-18 $[29]^{*}$ \\
\hline & & 11 & & OXCAP-MH [36] ${ }^{*}$ \\
\hline & & 12 & \multirow[t]{2}{*}{ CQ-CMH } & $\mathrm{CQ}-\mathrm{CMH}[37]^{*}$ \\
\hline & & 13 & & ACQ-CMH [38]* \\
\hline & & 14 & \multicolumn{2}{|c|}{ Capability-based questionnaire -well-being in patients with chronic pain [39]* } \\
\hline & & 15 & \multicolumn{2}{|c|}{ CADA $[40]^{*}$} \\
\hline
\end{tabular}

*Note: This table only indicates the articles reporting on the development of the respective tool. ICECAP-O ICEpop CAPability measure for older people, ICECAP- $A$ ICEpop CAPability measure for adults, ICECAP-FC ICEpop CAPability and Functioning measure, ICECAP-SCM ICEpop CAPability measure for supportive care, OCAP Oxford Capability Questionnaire, OXCAP-MH Oxford Capability Questionnaire for Mental Health, CQ-CMH Capability Questionnaire for Community Mental Health 


\section{Types of measurement instruments}

Table 2 provides an overview of the different measurement tools reported in the 52 identified articles. We found that instruments to assess capabilities fall into three major categories: (1) qualitative tools, e. g. using interviews or videography $(n=5),(2)$ quantitative tools, e. g. questionnaires $(n=46)$, and (3) mixed method approaches using a combination of interviews and questionnaires $(n=1)$.

In the quantitative category, $\mathrm{n}=5$ articles measured capabilities through analyzing secondary data (e. g. data from the British Panel Household Survey [41]), while $n=41$ covered a total of eleven individual questionnaires. Of these, four belong to the ICECAP-family (ICEpop CAPability index of the "Investigating Choice Experiments for the Preferences of Older People" project) and use varying sets of items to cover specific target groups and outcome variables: the ICECAP-O for older adults [31] and ICECAP-A for adults [32] with five items each, the ICECAP-SCM measuring capabilities of people in need of supportive care [33] containing seven items, and the ICECAP-FC for adults measuring both functioning and capabilities [34] with ten items. Another set of questionnaires comes from the "Oxford Capability Questionnaire" family, including the original OCAP (Oxford capability Questionnaire) [35] with 64 items, the shortened OCAP-18 [42] (18 items), and a version adapted to mental health, the OxCAP-MH (Oxford Capability Questionnaire for Mental Health) [36] (16 items). The most comprehensive questionnaires are the CQ-CMH (Capability Questionnaire for community mental health) [37] with 104 items and its adapted version, the $A C Q-C M H$ (Achieved Capability Questionnaire for community mental health) [38] with 98 items. The systematic search further identified two questionnaires that did not belong to a larger "family" of tools, the Capability Based Questionnaire for Patients with Chronic Pain [39] (8 items) and the Capability Assessment for Diet and Activity (CADA) geared at adults suffering from obesity and diabetes [40]. All identified questionnaires are constructed for self-completion and use subjective measures to assess capabilities.

\section{Main aims and methods employed}

Table 3 reports on the main aims of the included articles as well as the main methods used to develop the individual measurement tool or to empirically test its measurement properties. Out of the 52 included articles, 8 described the development of a measurement instrument [31-35, 39, 40, 42], 20 focused on checking psychometric properties of existing tools $[49,50,52,54-58$, $61,63-71,74], 2$ evaluated different instruments comparatively $[28,75]$, and 8 reported results of actual measurements of health-related capabilities [23, 24, 27, 41,
44-47]. The remaining $(n=14)$ articles had a mixed focus: on development/measurement $(n=2)$ [25, 26], development/psychometric properties $(n=9)[29,30$, 36-38, 43, 51, 53, 62], or comparison/psychometric properties $(n=3)[59,60,73]$.

Among the qualitative tools, only Sauter et al. [25] provided details on the development process: Their interview guidelines were the result of literature screening and a conscious selection of specific items from the $O C A P$ questionnaire [35]. The identified questionnaires were developed using different methodologies. For example, the OPCAP [35] is based on a set of largely theoretical criteria by Martha Nussbaum, who co-developed the original capability approach [76]. The ICECAP-O questionnaire was compiled based on a previously conducted literature review and developed through indepth interviews with the respective target group [31]. The ICECAP-A [32] and ICECAP-SCM [33], the Capability Based Questionnaire for Patients with Chronic Pain [39], and CADA [40] were developed by conducting iterative interviews with the respective target group. The CQ-CMH [37] emanated from the analysis of focus group data, expert opinion, and an additional alignment with the Nussbaum criteria.

Articles reporting on the validation of questionnaires used different methodological approaches. Convergent and construct validity were mostly investigated by correlating results with those measured via other questionnaires that measure well-being or health aspects (e. g. EQ-5D) $[29,35,36,38,51,53,55,58,60,62,66-68,70$, $73,74]$ or using Chi-Square analysis $[31,52,64]$. Discriminant validity was ascertained by performing uni- or multivariate analysis $[29,55,58,67-69,71,73]$. Some questionnaires were further been checked regarding their reliability using test-retest analysis $[29,38,43,57$, $62,73,74,77]$, or regarding their responsiveness via anchor-based analysis [54, 66, 67]. Moreover, the $I C E$ $C A P-O$ and the $O x-C A P$ questionnaire were evaluated comparatively to the EQ-ED questionnaire by correlating their results [59, 60, 73, 75].

Articles reporting on studies that directly measured capabilities without developing or validating any tools for future use were only found among the qualitative studies and secondary data analyses. Qualitative measurement was performed either by semi-structured interviews [23-26], observation [24] or video analysis [27], while secondary data was analyzed via methods such as regression [41] or equation modelling [44].

\section{Measurement properties}

The major measurement properties of the different tools are shown in Table 4. Sample sizes among the qualitative and mixed methods approaches varied between $n=$ 12 [27] and $n=64$ [26], while numbers were naturally 
Table 3 Description of included studies and tools

Qualitative tools

No. Tool

1. Interview

\section{No. Author (year) Study Focus of tool of country items}

$$
\text { (2014) [23], }
$$

$$
\text { Canada }
$$

n.a. Ndomoto et al. $\mathrm{M}$ (2018) [24], UK

10

Sauter et al.

(2018) [25],

Germany

D/M Health enhancing PA

Health and diabetes self-management

Health

enhancing PA

\section{6/21 Chakraborty} et al. (2020) [26]
D/M Healthy children's growth

region of
Bangladesh

2. Videography
n.a. Petros et al. (2016) [28], USA

Mental health recovery
Bangla/English

English

Language
tool
English
English

German/English living in

Adults with mental illness senior senior
residences

Adults with diabetes

Adults living in rural Kenya and urban deprived UK

Older adults

children living in hoar Bangladesh

\begin{tabular}{ll}
$\begin{array}{l}\text { Language of } \\
\text { tool }\end{array}$ & $\begin{array}{l}\text { Target } \\
\text { population }\end{array}$ \\
$\begin{array}{ll}\text { Luxembourgish, } & \text { Adult care } \\
\text { Portuguese, } & \text { givers } \\
\text { French, German } & \end{array}$ \\
\hline
\end{tabular}

$\begin{array}{llll}\begin{array}{l}\text { No. } \\ \text { of } \\ \text { items }\end{array} & \begin{array}{l}\text { Author (year) } \\ \text { country }\end{array} & \begin{array}{l}\text { Study } \\ \text { Aim }\end{array} & \text { Focus of Tool } \\ 20 & \begin{array}{l}\text { Bucki et al. } \\ \text { (2016) }[43],\end{array} & \text { C } & \text { Health } \\ \text { Luxembourg } & & \end{array}$

\section{Mixed method}

\section{No. Instrument}

3. Questionnaire and Interview

Quantitative tools

No. Instrument

$$
\begin{aligned}
& \text { No. Author (year) } \\
& \text { of country } \\
& \text { items }
\end{aligned}
$$

4. Questionnaires used in secondary data n.a.

Zaineh \& [44], France

\section{Study Focus of Tool Aim}

\section{$M$}

Health and selfmanagement

$\begin{array}{llll}\text { n.a. } & \begin{array}{l}\text { Anand et al. } \\ \text { (2005) [41], UK }\end{array} & & \text { General WB } \\ \text { n.a. } & \begin{array}{l}\text { Douptcheva } \\ \text { et al. (2014) } \\ \text { [45], UK }\end{array} & & \\ & & & \\ & \end{array}$

\section{Language of \\ tool \\ English \\ Target population \\ Young adults living in Palestine}

English

English

rench in British
Development of interview-guide by $R T$ based on Anand's capability questionnaire [35] and literature on older adult's physical activity; Measurement via semistructured interviews

Development of FG and individual interview guide by $R T$ based on literature review and pilot testing; Measurement via FG and in-depth interviews with parents

Four-week measurement via videography on the topic Tell us about your recovery; No RT present during recording; Transcription and analysis of data by $R T$

\section{Method}

Measurement of relations between health capability factors of care givers using questionnaire-based (HCFC-8) interviews. Statistical analysis using Monte Carlo Markov Chain algorithms.

\section{Method}

Measurement of capabilities (health awareness, knowledge and living conditions) via Exploratory Structural Equation Modelling using data from the Palestinian Family Survey.

Adults living Measurement of capabilities and well-being by regression using

households data of the British Household Panel Survey

Women Measurement of capabilities and living in functionings to identify factors Accra that influence our health using data from the Women's Health Study of Accra - Wave II.

Older adults Measurement of capabilities 
Table 3 Description of included studies and tools (Continued)

(2016) [46],

France
Subjective WB and Panek (2020) [47],

Poland

5. ICECAPI ICECAP-O
5

Coast et al. (2008) [31], UK

D

General WB

5

Coast et al. PP

(2008) [49], UK

General WB
Polish

(a)

English

$$
\text { (1) }
$$

English

Adults $\geq 65$

General
population $\geq$
16

$$
16
$$

Adults $\geq 65$

(n)

Adults $\geq 65$

(2011) [50], UK
5
Couzner et al.
(2012) [52],
Australia
5 Makai et al.
(2012) [53],

Netherlands

D/PP General WB

\section{5}

Davis et al.

(2013) [54],

Canada

5

Makai et al.

(2013) [55],

Netherlands
C/PP

General WB

PP

General WB

(1)


Table 3 Description of included studies and tools (Continued)

et al. (2017)

[58], Spain

5

Franklin et al, (2018) [59], UK

$\mathrm{C} / \mathrm{PP}$

General WB

English

Adults $\geq 65$

Milte et al.

C/PP General WB

(2018) [60],

Australia

5

Mitchell et al.

(2020) [61], UK

Baji et al. (2020) [62],

Hungary

Adult $\geq 65$

Hungarian $\quad$ Adult $\geq 65$
6.

ICECAP-A

5

(2012) [32], UK
Al-Janabi et al. D

General WB

(1)

$\begin{array}{llll}5 & \begin{array}{l}\text { Al-Janabi et al. PP } \\ \text { (2013) [63], UK }\end{array} & \text { General WB } & \text { English } \\ 5 & \begin{array}{l}\text { Al-Janabi et al. PP } \\ \text { (2013) [64], UK }\end{array} & \text { General WB } & \text { English } \\ 5 & \begin{array}{l}\text { Al-Janabi et al. PP } \\ \text { (2015) [65], UK }\end{array} & \text { General WB } & \text { English } \\ 5 & \begin{array}{l}\text { Keeley et al. PP } \\ \text { (2015) [66], UK }\end{array} & \text { General WB } & \text { English } \\ & \begin{array}{l}\text { Goranitis et al. PP } \\ \text { (2016) [67], UK }\end{array} & \text { General WB } & \text { English }\end{array}$

English

General WB English analysis) and convergent validity (correlation with dimensions of the EQ-5D + C, ADRQL, ADL), and reliability (internal consistencyCronbach Alpha)

Comparison of (1) tariff scores using OLS and CLAD regression models and (2) domain scores using $M N L$ regression against the EQ-5D-3L

Adults $\geq 65$ Comparison against the EQ-5D$3 \mathrm{~L}$ using Spearman correlation coefficient and multiple linear regression

Measurement of response validity among people requiring kidney care using a think-aloud study

$R T$ translated original version into Hungarian; forward-backward translation; interviews ( $n=15)$ to assess comprehensiveness and relevance; Measurement of: construct validity (one-way subgroup comparison and regression analysis); convergent validity (Pearson's and Spearman's correlation (with EQ-5D-5L, EQ VAS, WHO-5; happiness and satisfaction VAS, SWLS); Test-retest reliability (ICC baseline and $5 \%$ of participants right after baseline

Adults $\geq 18$

Identification of important components of life through indepth interviews; Iterative semistructured interviews to refine attributes to a self-completion measure with one item per attribute

Adults $\geq 18$ Think-aloud and semi-structured interviews to assess the feasibility of a self-reporting capability measurement

Adults $\geq 18 \quad$ Measurement of construct validity (univariate analysis and correlations based on hypotheses made in advance)

Adults $\geq 18$ Measurement of test-retest reliability (ICC- baseline and 2-week capability index scores)

Adults $\geq 18$ Measurement of responsiveness (anchor-based analysis; anchors: EQ-5D-3L, GAD-7, PHQ-8)

Adults $\geq 18$ Measure of acceptability, construct validity (convergent: Pearson's correlation with EQ-5D$3 \mathrm{~L}$ and ICIQ-OAB, Spearman's correlation coefficient across dimension scores, and index and dimension scores; discriminant: one-way ANOVA and KruskalWallis $H$ test)

Adults $\geq 18 \quad$ Assessment of construct validity (convergent: Pearson's 
Table 3 Description of included studies and tools (Continued)

\begin{tabular}{|c|c|c|c|c|c|c|}
\hline 5 & $\begin{array}{l}\text { Mitchell et al. } \\
\text { (2017) [69], UK }\end{array}$ & PP & General WB & English & Adults $\geq 18$ & $\begin{array}{l}\text { Concept-mapping from } \\
\text { condition-specific and capability } \\
\text { items; Discriminant validity test- } \\
\text { ing (Mann-Whitney U test using } \\
\text { DASS-D and K10 data; Multivari- } \\
\text { able regression analysis using } \\
\text { OLS) }\end{array}$ \\
\hline 5 & $\begin{array}{l}\text { Linton et al. } \\
\text { (2018) [70], } \\
\text { Germany }\end{array}$ & PP & General WB & German & Adults $\geq 18$ & $\begin{array}{l}\text { Measurement of internal- } \\
\text { consistency (Cronbach's Alpha), } \\
\text { convergent (Pearson's correlation } \\
\text { with EQ-5D-3L, SF-6D, SWLS } \\
\text { scores), and construct validity } \\
\text { (OLS regressions) }\end{array}$ \\
\hline 5 & $\begin{array}{l}\text { Tang et al. } \\
\text { (2018) [51], } \\
\text { China }\end{array}$ & D/PP & General WB & Chinese & Adults $\geq 18$ & $\begin{array}{l}R T \text { translated original version into } \\
\text { Chinese; FG evaluated } \\
\text { appropriateness of the } \\
\text { translation; pilot testing; } \\
\text { backward translation; online- } \\
\text { survey to check acceptability, re- } \\
\text { liability (item correlations), and } \\
\text { validity (EFA and correlations } \\
\text { with EQ-5D-3L and EQ-VAS) }\end{array}$ \\
\hline 5 & $\begin{array}{l}\text { Holst- } \\
\text { Kristensen } \\
\text { et al. (2020) } \\
\text { [43], Denmark }\end{array}$ & $\mathrm{D} / \mathrm{PP}$ & General WB & Danish & Adult $\geq 18$ & $\begin{array}{l}R T \text { translated original version into } \\
\text { Danish; forward-backward trans- } \\
\text { lation; pilot-testing in general } \\
\text { population }\end{array}$ \\
\hline 5 & $\begin{array}{l}\text { Shahataheri } \\
\text { et al. (2020) } \\
\text { [29], Iran }\end{array}$ & $\mathrm{D} / \mathrm{PP}$ & General WB & Persian & Adult $\geq 18$ & $\begin{array}{l}R T \text { translated original version into } \\
\text { Persian; forward-backward trans- } \\
\text { lation; pilot-testing in general } \\
\text { population; Measurement of test- } \\
\text { retest reliability (ICC-baseline and } \\
\text { 2-week) }\end{array}$ \\
\hline 5 & $\begin{array}{l}\text { Mah et al. } \\
\text { (2020) [71], } \\
\text { Canada }\end{array}$ & PP & General WB & English & Adult $\geq 18$ & $\begin{array}{l}\text { Measurement of construct } \\
\text { validity: discriminant ( } t \text { test, linear } \\
\text { trend analysis or multiple } \\
\text { regression); convergent } \\
\text { (correlation with measures of the } \\
\text { same concept: AQoL-8D, EQ-5D- } \\
5 \text { L, HUl-3, SF- 6D) }\end{array}$ \\
\hline 5 & $\begin{array}{l}\text { Mitchell et al. } \\
\text { (2020) [61], UK }\end{array}$ & PP & General WB & English & Adult $\geq 18$ & $\begin{array}{l}\text { Measurement of response } \\
\text { validity among people requiring } \\
\text { kidney care using a think-aloud } \\
\text { study }\end{array}$ \\
\hline 5 & $\begin{array}{l}\text { Baji et al. } \\
\text { (2020) [62], } \\
\text { Hungary }\end{array}$ & D/PP & General WB & Hungarian & Adult $\geq 18$ & $\begin{array}{l}R T \text { translated original version into } \\
\text { Hungarian; forward-backward } \\
\text { translation; interviews }(n=15) \text { to } \\
\text { assess comprehensiveness and } \\
\text { relevance; Measurement of: con- } \\
\text { struct validity (one-way subgroup } \\
\text { comparison and regression ana- } \\
\text { lysis); convergent validity (Pear- } \\
\text { son's and Spearman's correlation } \\
\text { (with EQ-5D-5L, EQ VAS, WHO-5; } \\
\text { happiness and satisfaction VAS, } \\
\text { SWLS); Test-retest reliability (ICC } \\
\text { baseline and 5\% of participants } \\
\text { right after baseline }\end{array}$ \\
\hline 7 & $\begin{array}{l}\text { Sutton \& Coast } \\
\text { (2014) [33], UK }\end{array}$ & $\mathrm{D}$ & WB in end of life care & English & $\begin{array}{l}\text { People at } \\
\text { end of life }\end{array}$ & $\begin{array}{l}\text { Interviews to determine } \\
\text { conceptual elements of a good } \\
\text { death; follow-up interviews to } \\
\text { check conceptual attributes }\end{array}$ \\
\hline
\end{tabular}

correlation with EQ-5D-5L; Discriminant: univariate and multivariate analysis) and sensitivity to change

cept-mapping from ing (Mann-Whitney $U$ test using DASS-D and K10 data; Multivariable regression analysis using

Measurement of internalwith EQ-5D-3L, SF-6D, SWLS scores), and construct validity

$R T$ translated original version into backward translation; onlinesurvey to check acceptability, revalidity (EFA and correlations $R T$ translated original version into Danish; forward-backward transation; pilot-testing in general $R T$ translated original version into Perward-backward tran population; Measurement of testretest reliability (ICC-baseline and Measurement of construct trend analysis or multiple regression); convergent ,

Measurement of response kidney care using a think-aloud $R T$ translated original version into Hungarian; forward-backward ranslation; interviews $(n=15)$ to and struct validity (one-way subgroup comparison and regression anaconvergent validity (Pearwith EQ-5D-5L, EQ VAS, WHO-5: (with EQ-5D-5L, EQ VAS, WHO-5; baseline and $5 \%$ of participants check conceptual attributes 
Table 3 Description of included studies and tools (Continued)

\begin{tabular}{|c|c|c|c|c|c|c|c|c|}
\hline 8. & ICECAP-FC & 10 & $\begin{array}{l}\text { Al-Janabi } \\
\text { (2018) [34], UK }\end{array}$ & $D$ & $\begin{array}{l}\text { WB capabilities and } \\
\text { functionings }\end{array}$ & English & Adults $\geq 18$ & $\begin{array}{l}\text { ICECAP-A modified with } \\
\text { additional question on } \\
\text { functioning to each attribute by } \\
\text { RT }\end{array}$ \\
\hline 9. & OCAP & 64 & $\begin{array}{l}\text { Anand et al. } \\
\text { (2009) [35], UK }\end{array}$ & $D$ & $\begin{array}{l}\text { General Capabilities (e.g. } \\
\text { enjoying recreational } \\
\text { time, political views, } \\
\text { making friends bodily } \\
\text { health and integrity) }\end{array}$ & English & Adults $\geq 18$ & $\begin{array}{l}\text { Development of items based on } \\
\text { Nussbaum criteria [72] }\end{array}$ \\
\hline 10. & OCAP-18 & 18 & $\begin{array}{l}\text { Lorgelly et al. } \\
\text { (2015) [42], UK }\end{array}$ & $D$ & $\begin{array}{l}\text { General Capabilities (e.g. } \\
\text { enjoying recreational } \\
\text { time, political views, } \\
\text { making friends bodily } \\
\text { health and integrity) }\end{array}$ & English & Adults $\geq 18$ & $\begin{array}{l}\text { Items, based on OCAP- } \\
\text { questionnaire [35], reduced on } \\
\text { analysis of } F G \text {, cognitive inter- } \\
\text { views, and factor analysis }\end{array}$ \\
\hline \multirow[t]{4}{*}{11.} & OxCAP-MH & 16 & $\begin{array}{l}\text { Simon et al. } \\
\text { (2013) [36], UK }\end{array}$ & $\mathrm{D} / \mathrm{PP}$ & $\begin{array}{l}\text { General capabilities for } \\
\text { mental health }\end{array}$ & English & $\begin{array}{l}\text { Adults } \geq 18 \\
\text { with a } \\
\text { mental } \\
\text { illness }\end{array}$ & $\begin{array}{l}\text { Adaption of the OCAP-18 [20] } \\
\text { based on expert-FG and valid- } \\
\text { ation (correlation with GAF, EQ- } \\
\text { 5D-VAS, EQ-5D-3L) }\end{array}$ \\
\hline & & 16 & $\begin{array}{l}\text { Vergunst et al. } \\
\text { (2017) [49], UK }\end{array}$ & PP & $\begin{array}{l}\text { General capabilities for } \\
\text { mental health }\end{array}$ & English & $\begin{array}{l}\text { Adults } \geq 18 \\
\text { with a } \\
\text { mental } \\
\text { illness }\end{array}$ & $\begin{array}{l}\text { Measurement of internal- } \\
\text { consistency (Cronbach's alpha), } \\
\text { test-retest (1-week apart; ICC), } \\
\text { and construct validity (correlation } \\
\text { with EQ-5D, BPRS, GAS, SIX) }\end{array}$ \\
\hline & & 16 & $\begin{array}{l}\text { Simon et al. } \\
\text { (2018) [30], UK }\end{array}$ & $\mathrm{D} / \mathrm{PP}$ & $\begin{array}{l}\text { General capabilities for } \\
\text { mental health }\end{array}$ & English & $\begin{array}{l}\text { Adults } \geq 18 \\
\text { with a } \\
\text { mental } \\
\text { illness }\end{array}$ & $\begin{array}{l}\text { Forward-backward-translation of } \\
\text { OxCAP-MH into German and lin- } \\
\text { guistic validation through Ger- } \\
\text { man native speakers }\end{array}$ \\
\hline & & 16 & $\begin{array}{l}\text { Laszewska } \\
\text { et al. (2019) } \\
\text { [73], Austria }\end{array}$ & C/PP & $\begin{array}{l}\text { General capabilities for } \\
\text { mental health }\end{array}$ & German & $\begin{array}{l}\text { Adults } \geq 18 \\
\text { with a } \\
\text { mental } \\
\text { illness }\end{array}$ & $\begin{array}{l}\text { Comparison against the EQ-5D- } \\
5 \mathrm{~L} \text { (EFA). Measurement of re- } \\
\text { sponsiveness (anchor question- } \\
\text { naires and standardized response } \\
\text { mean), discriminant validity (sub- } \\
\text { group comparison using } t \text { test } \\
\text { and one-way ANOVA), and test- } \\
\text { retest (ICC; baseline - max } 30 \\
\text { days after) }\end{array}$ \\
\hline 12. & $\mathrm{CQ}-\mathrm{CMH}$ & 104 & $\begin{array}{l}\text { Sacchetto et al. } \\
\text { (2016) [37], } \\
\text { Portugal }\end{array}$ & $\mathrm{D} / \mathrm{PP}$ & Mental Health & Portuguese & $\begin{array}{l}\text { Consumers } \\
\text { of mental } \\
\text { health } \\
\text { services }\end{array}$ & $\begin{array}{l}\text { FG interview data analysis; } \\
\text { development of item/rating } \\
\text { scale by steering committee and } \\
\text { additional comparison with } \\
\text { Nussbaum criteria [34]; } \\
\text { Assessment of face-validity }\end{array}$ \\
\hline 13. & ACQ-CMH-98 & 98 & $\begin{array}{l}\text { Sacchetto et al. } \\
\text { (2018) [38], } \\
\text { Portugal }\end{array}$ & $\mathrm{D} / \mathrm{PP}$ & Mental Health & Portuguese & $\begin{array}{l}\text { Consumers } \\
\text { of mental } \\
\text { health } \\
\text { services }\end{array}$ & $\begin{array}{l}\text { Adaption of the CQ-CMH ques- } \\
\text { tionnaire [37] based on panel } \\
\text { members judgement; Measure- } \\
\text { ment of validity (correlation with } \\
\text { WHOQOL-Bref, RAS, K6) }\end{array}$ \\
\hline 14. & $\begin{array}{l}\text { Capability- } \\
\text { based } \\
\text { questionnaire }\end{array}$ & 8 & $\begin{array}{l}\text { Kinghorn et al. } \\
\text { (2015) [39], UK }\end{array}$ & $D$ & WB & English & $\begin{array}{l}\text { People } \\
\text { suffering } \\
\text { from chronic } \\
\text { pain }\end{array}$ & $\begin{array}{l}\text { FG interview and individual } \\
\text { interviews to identify list of } \\
\text { important capabilities; } \\
\text { Development of questionnaire } \\
\text { for self-completion based on } \\
\text { identified capabilities by } R T\end{array}$ \\
\hline 15. & CADA & 34 & $\begin{array}{l}\text { Ferrer et al. } \\
\text { (2014) [40], } \\
\text { USA }\end{array}$ & $D$ & $\begin{array}{l}\text { Physical Activity and } \\
\text { Diet }\end{array}$ & English & $\begin{array}{l}\text { Adults with } \\
\text { obesity and } \\
\text { diabetes }\end{array}$ & $\begin{array}{l}\text { FG interviews were used to } \\
\text { identify important themes; } \\
\text { questionnaire created by } R T \\
\text { based on themes }\end{array}$ \\
\hline
\end{tabular}

ADL activities of daily living, ADRQL Alzheimer's disease related Quality of life, BPRS Brief Psychiatric Rating Scale, C Comparison, CTM-3 3-Item Care Transition Measure, $D$ Development, EFA exploratory factor analysis, FG Focus group; GAF Global Assessment of Functioning, ICC Intra-class correlation coefficient, $M$ 


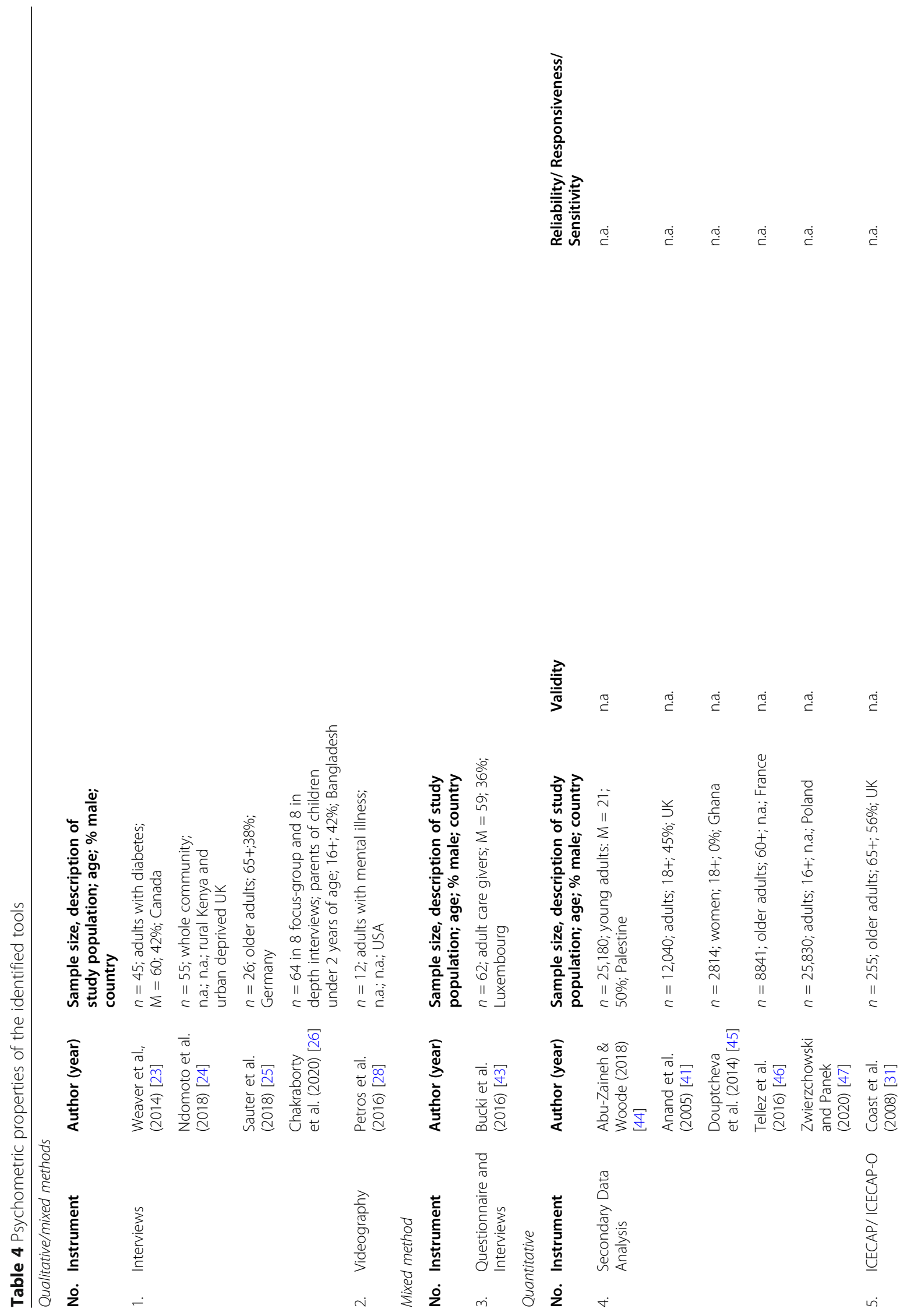




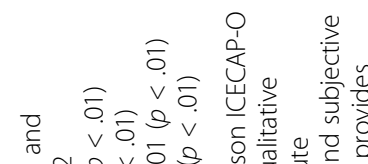

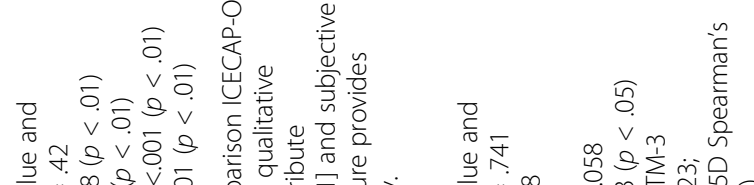

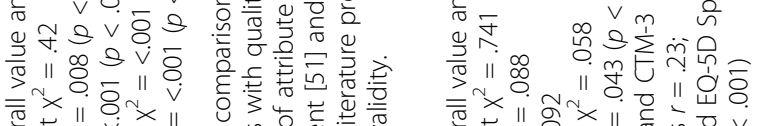
ثुँ

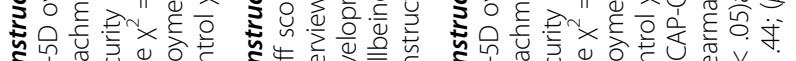

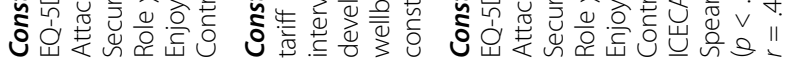
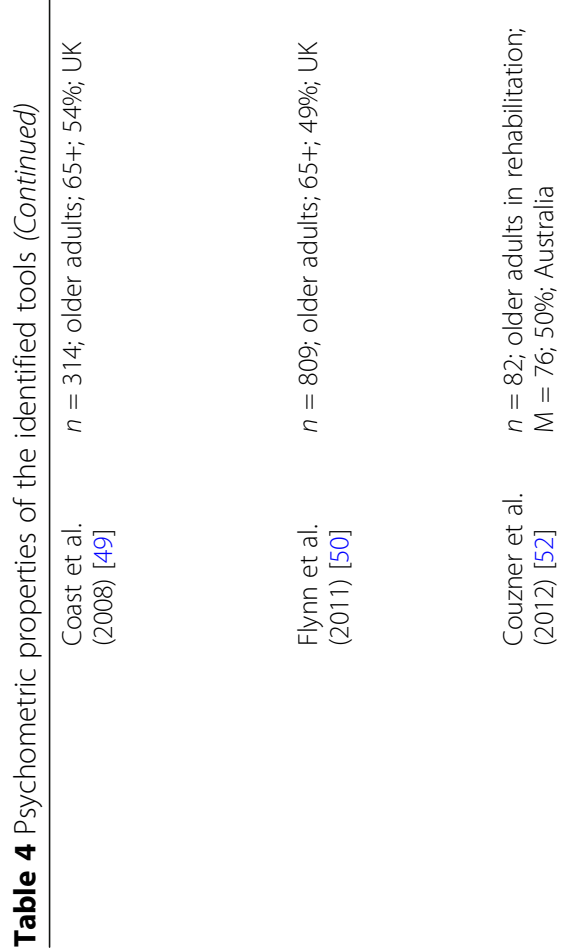

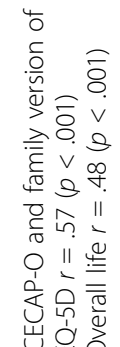

$$
\begin{aligned}
& \text { oิ }
\end{aligned}
$$

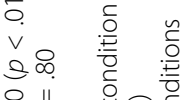

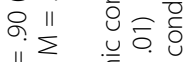

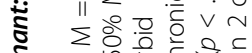

है

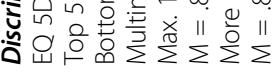
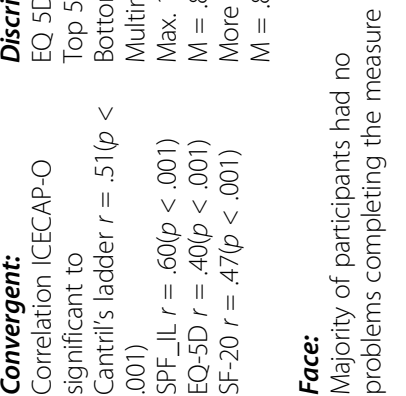

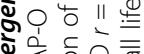

)

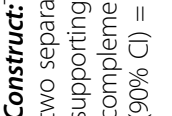

岂出。㐫

ตั้. 늠 는

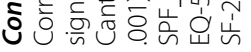
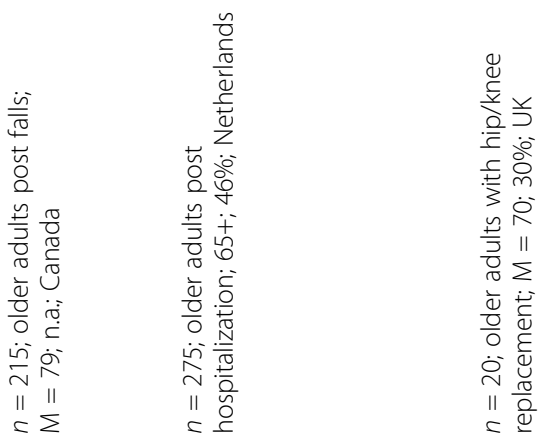

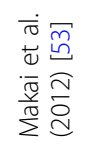

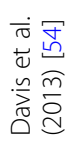

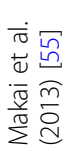

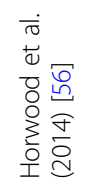




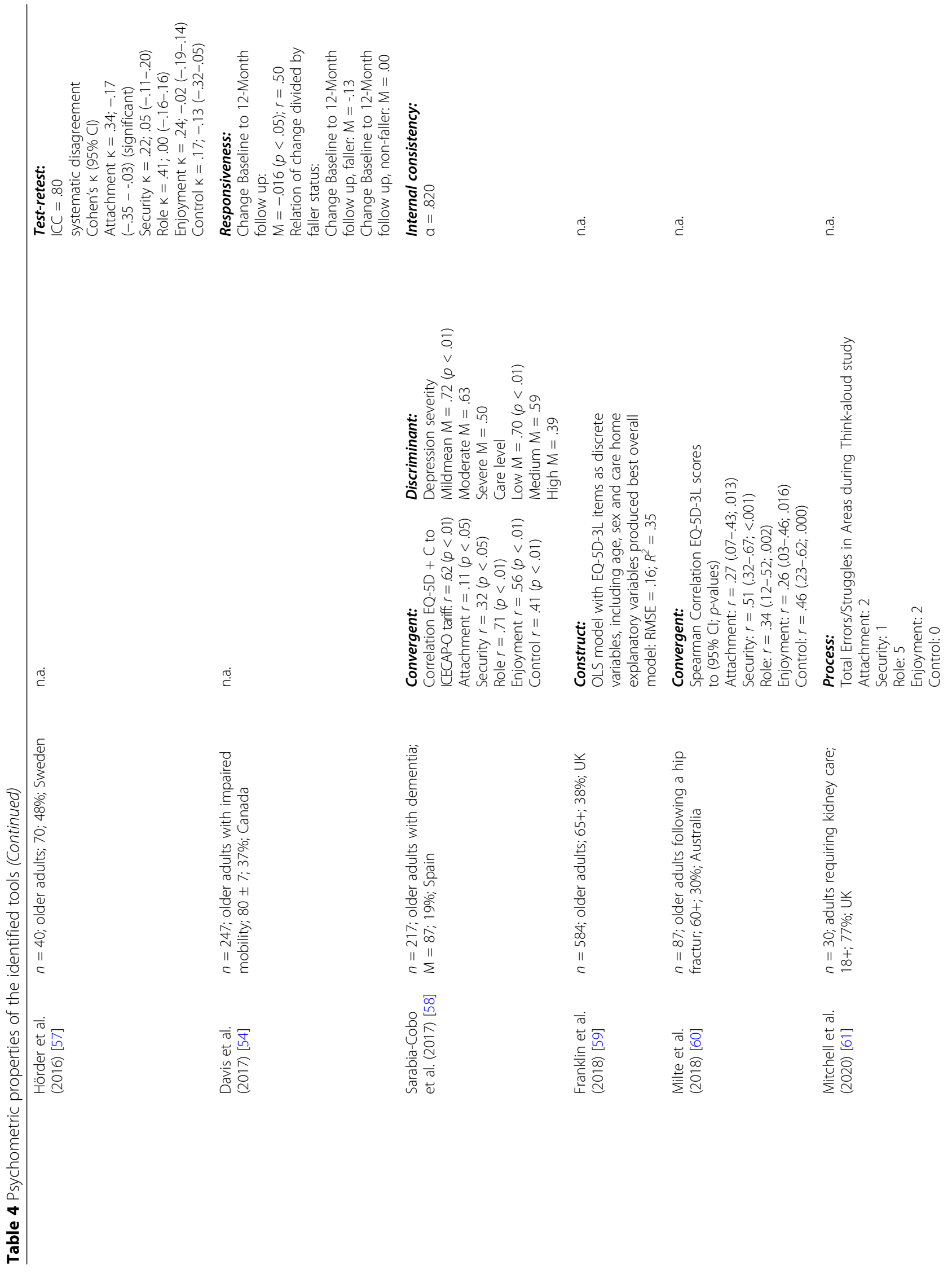



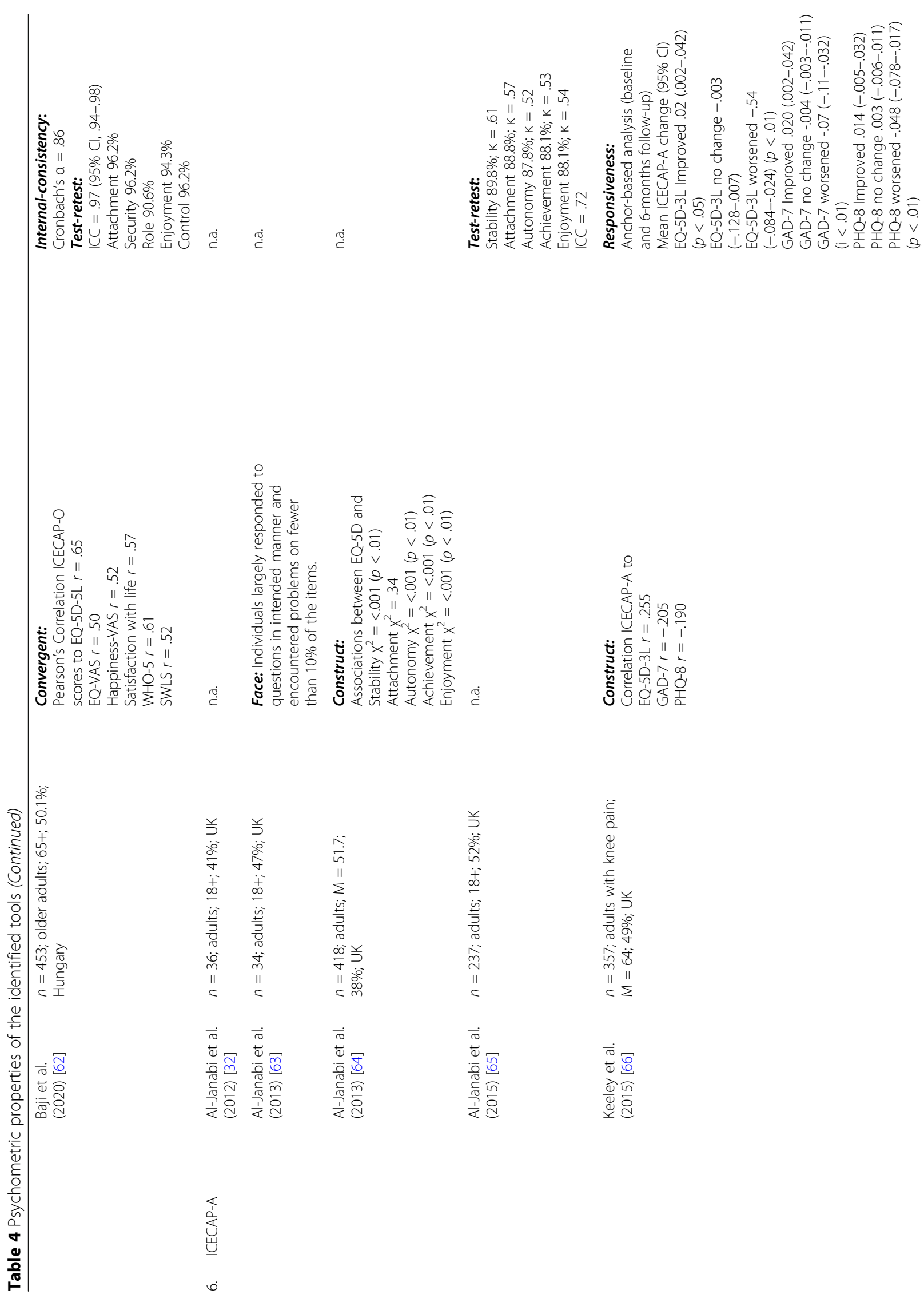

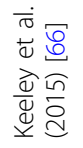



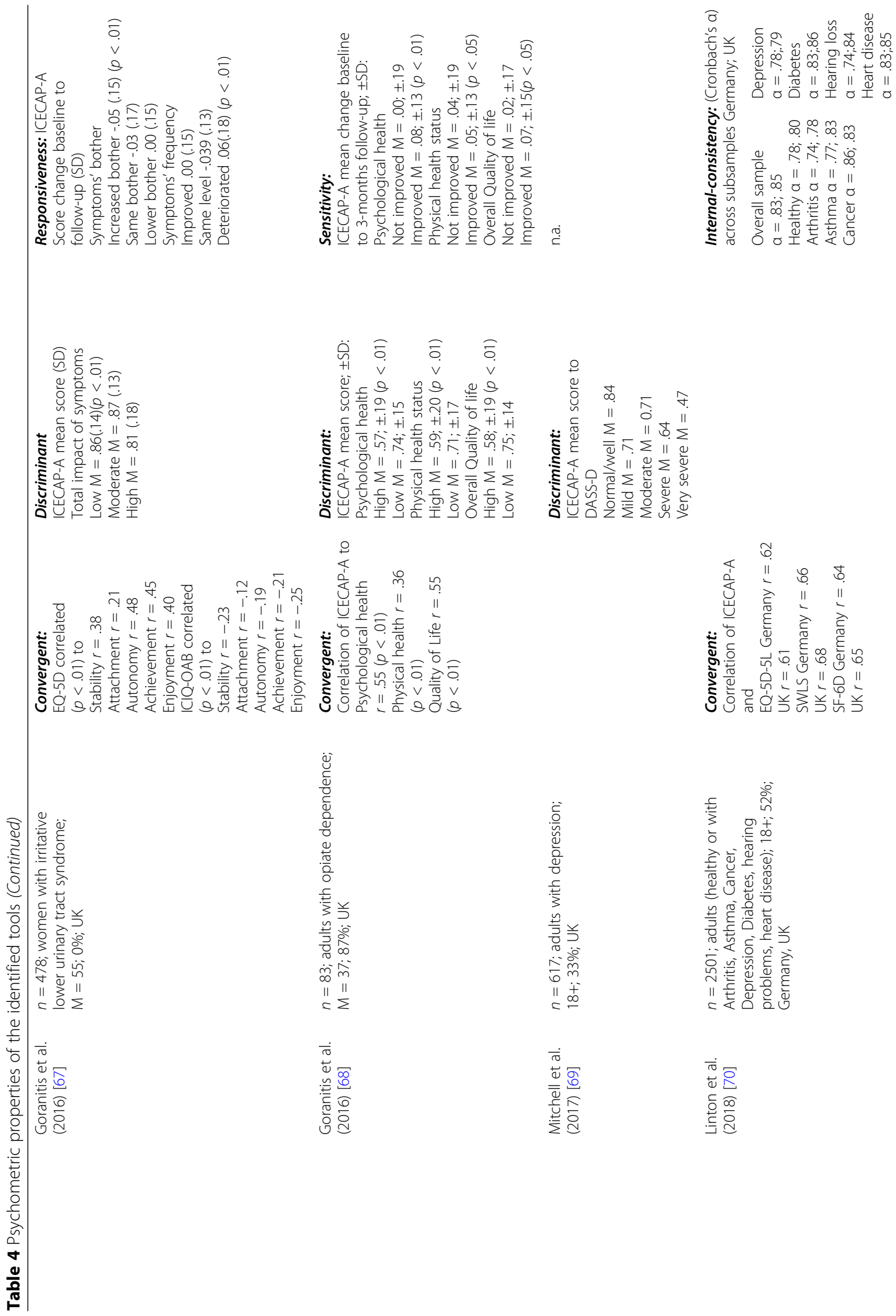

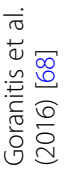
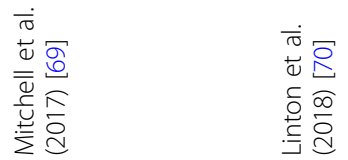


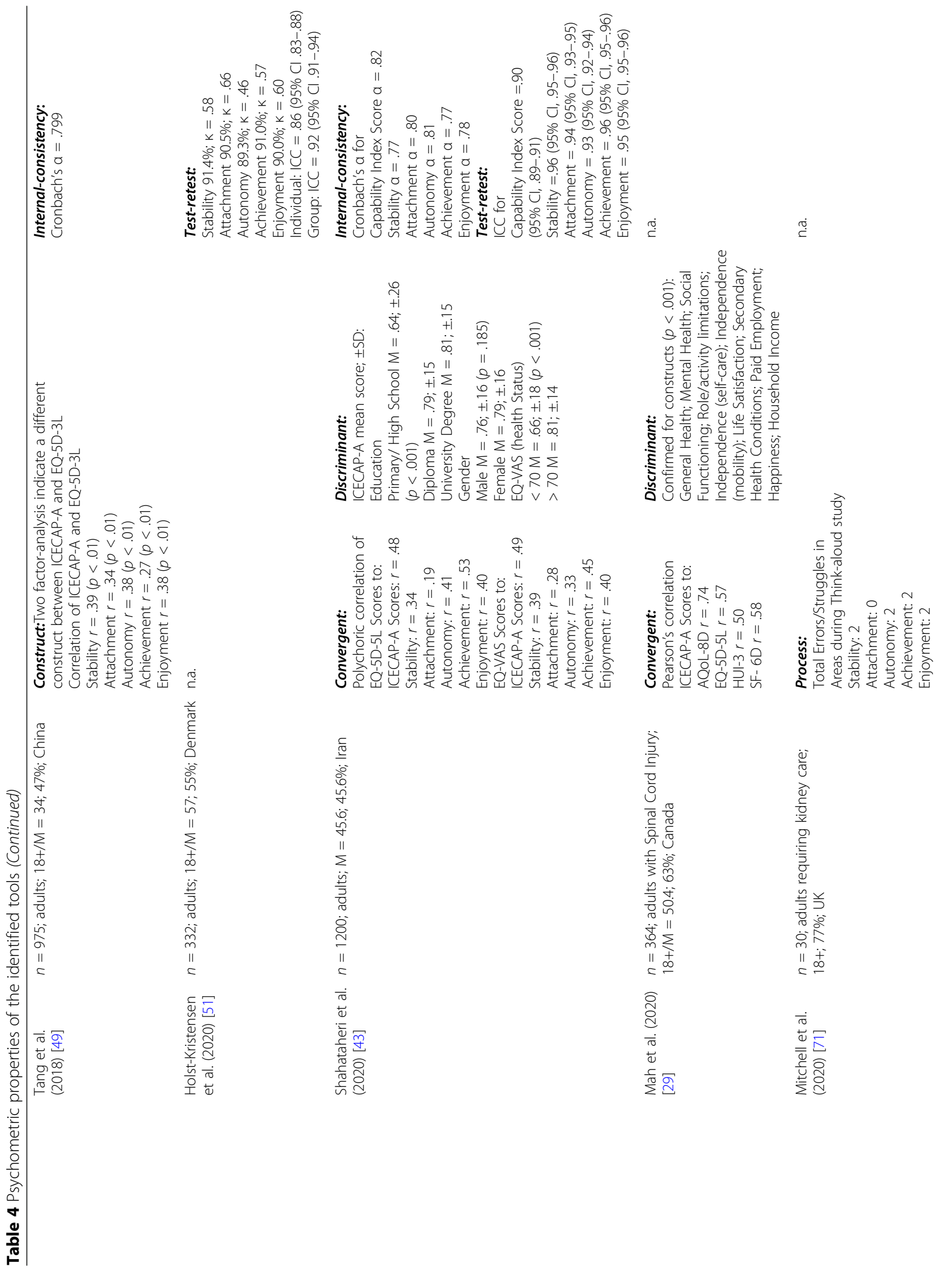



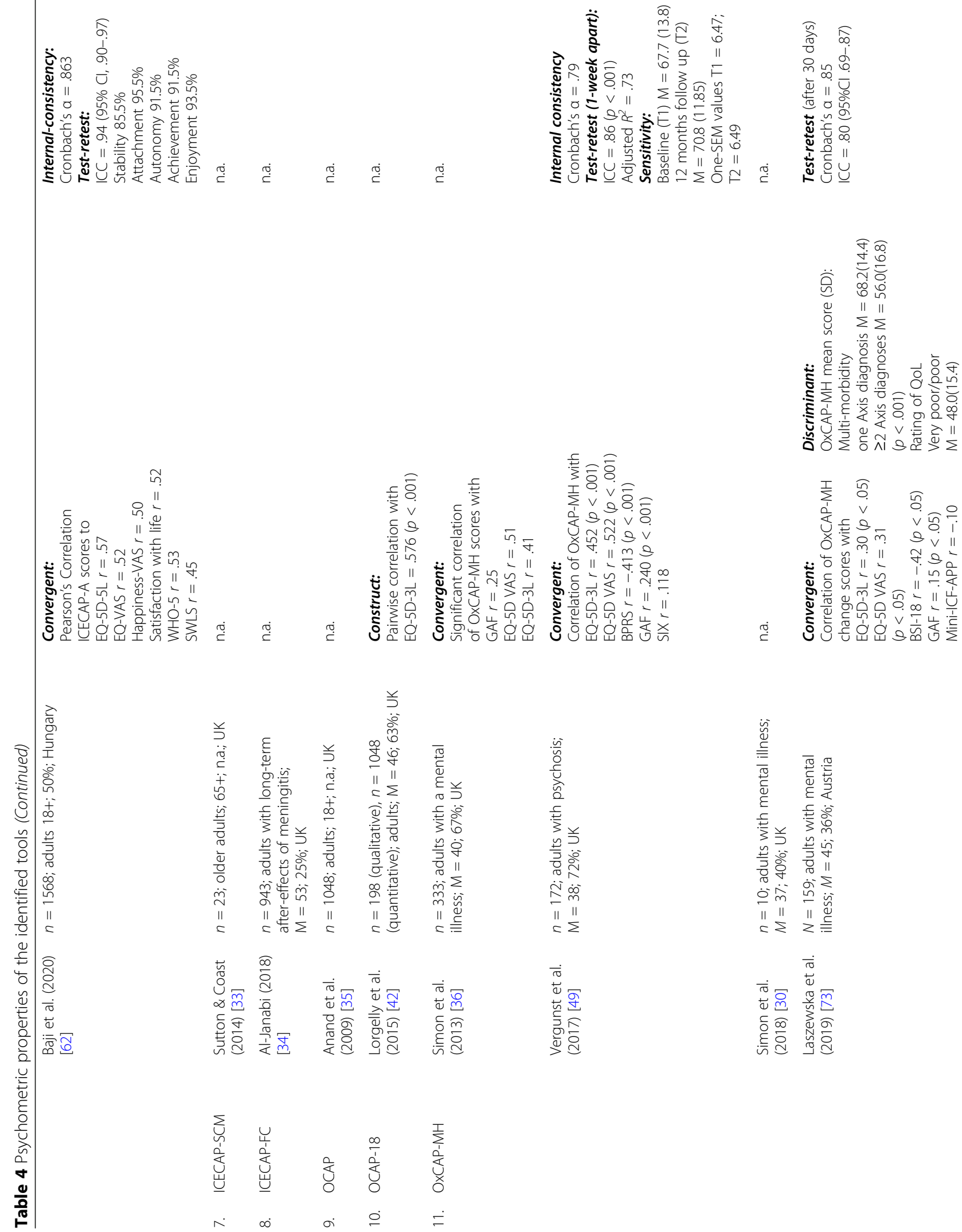

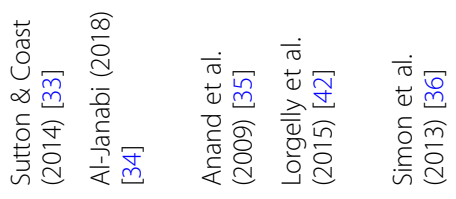
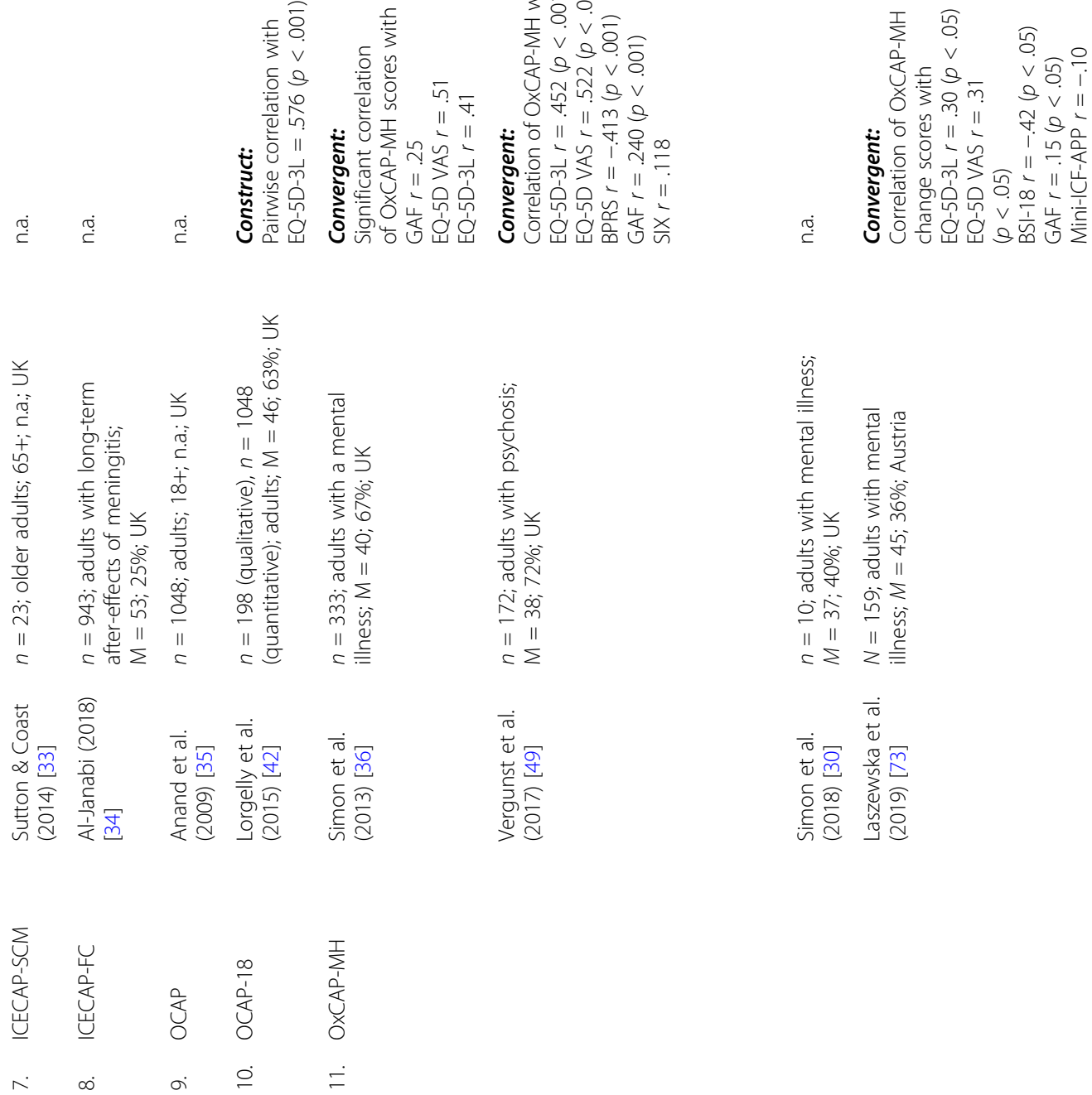


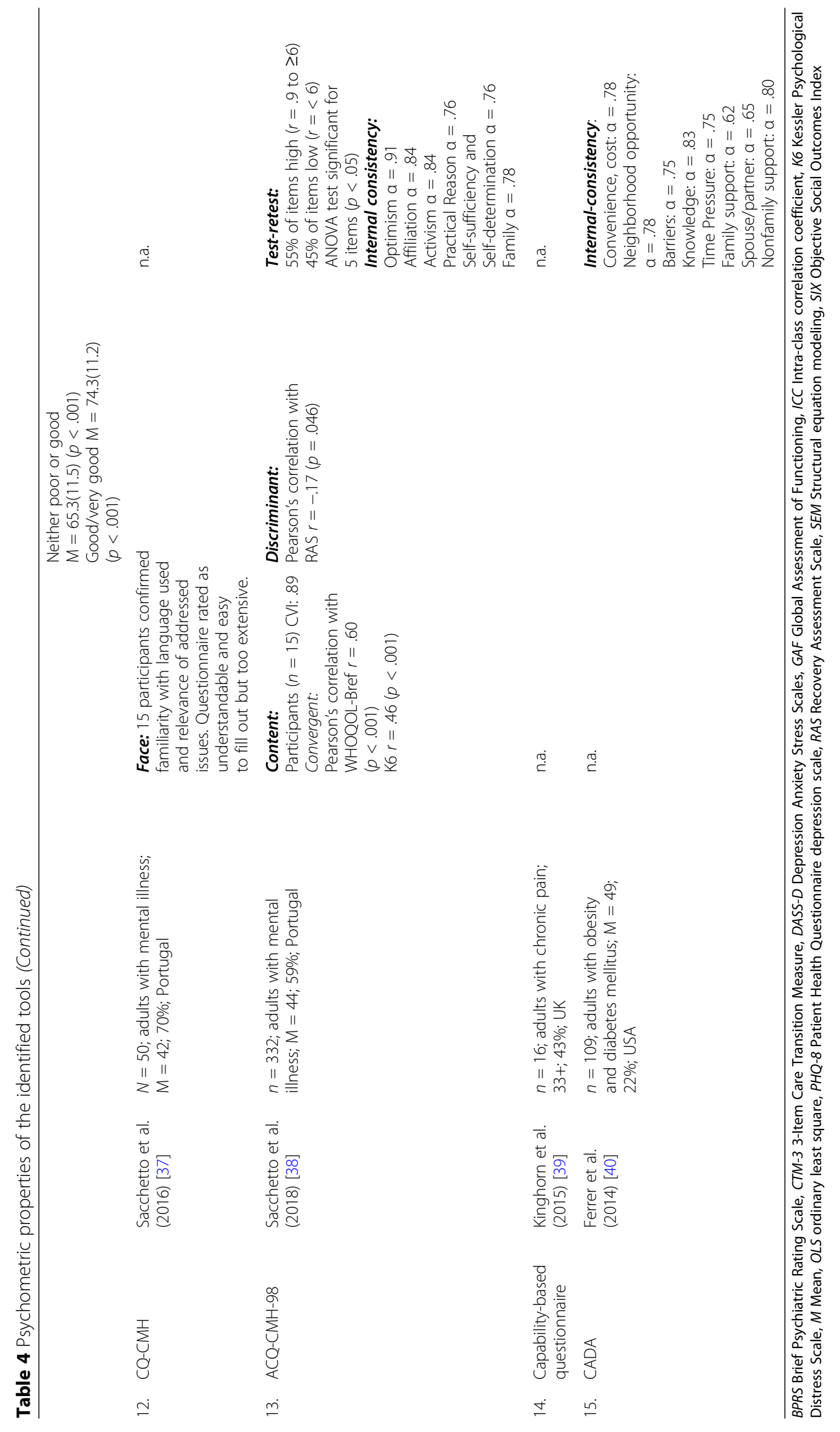


much larger for the secondary data analyses (between $n=$ 2814 [45] and $n=25,180$ [44]). Target groups varied widely, from adults in general [24, 32, 35, 41, 78], children under the age of two [26], young adults [44] and older adults $[25,31,46]$, to adults with special conditions or characteristics [23, 27, 36-40] or only women [45].

Sample sizes for the identified questionnaires varied substantially, ranging from $n=10$ [30] to $n=2501$ [70]. For the ICECAP-O, six articles reported on the intended target group of adults over $65[49,50,53,57,59,62]$. Other publications also applied it specifically to people with a medical condition $[54,58,61]$ or within a rehabilitation context $[52,55,56,60]$. The ICECAP-A was developed to measure capabilities among adults over 18. Six of the identified articles used this target group [29, 32, 43, $51,63,64,77]$ while others validated it pointedly for adults with knee pain [66], opiate dependence [68], depression [69], among women suffering irritative lower urinary tract syndrome [67], or adults with a spinal cord injury [71].

Detailed psychometric properties were only reported for the quantitative measurement instruments. The most detailed results were available for questionnaires of the ICECAP-family. Both the ICECAP-O and the ICECAP-A were reported to have good construct $[49,51,52,64,66]$, convergent $[29,53,55,58,60,62,67,70,71]$ validity when compared to the EQ-5D instrument to measure generic health status, and discriminant validity $[29,55,58,67,71]$. The ICECAP-O and ICECAP-A further showed good testretest reliability [29, 43, 57, 62, 77] and good internal consistency $[29,51,58,70]$. In addition, the ICECAP-A was also found to be significantly responsive among adults with knee pain [66] and women with irritative lower urinary tract syndrome [67]. No psychometric properties were reported for the ICECAP-SCM, ICECAP-FC questionnaires. In the $O C A P$ family, no details were available for the originally developed questionnaire [35]. The OCAP-18 only yielded good construct validity when correlated with the EQ-5D-3L questionnaire [42]. The adaption of the OCAP for mental health showed good convergent validity [36, 74], internal consistency, and test-retest reliability [74], which was also confirmed for its German version [73]. With respect to the other questionnaires, Sacchetto et al. [38] reported good content and discriminant validity as well as internal consistency for the $A C Q-C M H$. The $C A D A$ questionnaire [40] reported good internal consistency for most questions, while the Capability Measurement Tool for People with Chronic Pain [39] did not report any psychometric properties.

\section{Overall capabilities, capabilities for health, and capabilities for PA}

While some of the questionnaires focus on the overall capabilities to pursue one's goals and being content with one's own life (e. g. the ICECAP questionnaires [31-34]), others are concerned with more specific aspects, such as enjoying recreational time, political views, making friends, or areas relevant to this study, e. g. bodily health and integrity (e. g. OCAP questionnaires [35, 42, 74]). Some questionnaires focus on specific subsets of health enhancing factors, such as the CADA [40], which is concerned with capabilities for healthy diet and PA but does not measure overall capabilities for health or well-being. A similar pattern can be found for the qualitative tools: While Ndomoto et al. [24] focus on general capabilities for health, Abu-Zaineh et al. [44] explicitly deal with capabilities for health and self-management diabetes patients. Sauter et al. [25] is the only qualitative tool with a focus on capabilities for PA as a health-promoting factor.

Among the questionnaires, CADA [40] is the only one to directly measure capabilities for PA by specifically asking about resources (e.g. money to afford going to the gym) as well as environmental (e.g. indoor and outdoor PA spaces available), social (e.g. surrounding people are supportive of one's PA) and individual (e.g. mental and physical health influencing PA) factors of influence. The other questionnaires do not specifically ask for capabilities to pursue PA or sports but at least partially address areas that can be considered relevant for health-enhancing PA, such as physical suffering (ICECAP-SCM [33]), bodily health or enjoyment of recreational activities (OCAP [35] and OCAP-18 [42]). The qualitative tools do not explicitly address capabilities for PA. The only exception is Sauter et al. [25], which specifically asks for the individual (e. g. knowledge about PA), social (e. g. family and friends support) and environmental factors (e. g. offerings) that influence the opportunities of seniors in retirement homes to be physically active.

\section{Discussion}

The aim of this review has been to give an overview of the current state of research on available tools to measure capabilities for health based on the approach originally developed by Sen and Nussbaum, with a special focus on identifying those potentially relevant for HEPA. The systematic search was able to identify capability measurement tools for health and HEPA using qualitative, quantitative, and mixed methods between 2008 and 2020. It has explored the main features and psychometric properties of the identified tools, as well as their past application to different age and target groups.

Despite the number of papers identified, it is interesting to note that the number of distinct tools reported remains limited. For instance, there is a total of eleven questionnaire-based tools, most of which are variations and adaptations of either the ICECAP or the OPAC questionnaire. It is noteworthy that, although there are variations of the above-mentioned questionnaires for the 
use among different target groups, there is no tool available to objectively and comprehensively measure all aspects of health-related capabilities, especially when considering that the approach was first published in 1985 [1], connected to well-being as early as 1993 [3], and has recently gained even more attention in the field of public health.

The analysis revealed a great degree of methodological variation regarding the development of the interview guidelines and questionnaires. Some studies approached the development from a more philosophical view and based their interview guideline [25] or questionnaire items [35] on Martha Nussbaum's capability criteria [72]; others used an explorative approach, conducting focus-group [39, 40] or key-expert interviews $[36,42]$ to inductively develop their questionnaire. Another research group developed the questionnaire based solely on expert-group's opinion [38]. While our results allow no conclusions about which method is more appropriate or valid, those choosing a tool for a specific health promotion project should consider whether its development method and target group fit the intended application context. The variety of the available tools suggest that measuring capabilities may generally be a rather contextand target group-specific undertaking and may always require adaptation to different contexts and target groups. However, as this impedes the comparability of studies that target capabilities for health, working towards the development of tools applicable to more than one context may seem necessary.

The analyzed questionnaires that were empirically tested showed a moderate to good validity, reliability and responsiveness among different groups and compared to other questionnaires, mostly variations of the EQ-5D well-being questionnaire (i. e. EQ-5D-3L). This approach, however, poses an important theoretical issue, as it seems to imply that capability measures are better if they have a higher degree of correlation to measures of well-being. But according to Sen, well-being is a combination of "achieved functionings" [3], which are linked to but by no means perfectly correlated to a person's options (capabilities). To give an example, a person with a variety of options that may positively influence their health has the freedom to choose their eventual course of action and may actively decide not to realize a specific behavior. If we take the capability approach seriously, we must necessarily expect a considerable mismatch between functionings and capabilities and using this kind of validation approach appears as generally problematic. To validate such a measurement tool, a more comprehensive and thus perhaps more challenging approach might be necessary, e. g. by attempting to account for all individual, structural, and environmental opportunities as well as a target group's resources to validate the instrument.

Another issue is that the number of items used to measure capabilities also varied considerably between questionnaires, i. e. between five items (ICECAP-O/ ICECAP-A) and 104 items (OCAP). This raises the question whether all identified tools - even though they may have been validated - allow for measuring with the same accuracy. More research is required to investigate this, but in any case, health promoters interested in measuring capabilities will have to consider whether it will be feasible to administer the tool of their choice in practice, especially regarding those with a large number of items.

Most questionnaires were developed for a specific population group, e. g. adults (ICECAP-A [32], OCAP/ OCAP-18 [35, 42], CADA [40]), older adults (ICECAP-O [31]) or people suffering from mental illnesses (OxCAP$M H$ [36]; CQ-CMH [37]). However, even the general population questionnaires were often validated using samples of vulnerable population groups (e. g. adults with dementia [58], diabetes and obesity [40], or post hospitalization [55]). This may have implications for both the applicability and validity of the results for the general populace.

Our findings seem to support the conclusions of a previous literature review by Helter et al. [7] that there remain important conceptual and methodological issues in the field of measuring capabilities. At the same time, our study adds a new perspective, as Helter et al. [7] investigated the use of tools for economic evaluation while our main focus has been on measuring change and health intervention effectiveness.

Our research was guided by the intention to identify suitable tools for measuring capabilities for PA across the life-course. However, only two of the identified measurement instruments explicitly address PA, i. e. the $C A D A$ questionnaire [40] and the interview-based tool by Sauter et al. [25]. However, $C A D A$ is not geared exclusively at PA but combines it with capabilities for healthy diet. In addition, it was developed for populations suffering from obesity rather than general populations. Similarly, Sauter et al.'s tool has a specific focus on senior citizens. In other questionnaires, only individual items might be considered relevant for PA, e. g. questions on bodily health $[35,36,42]$. Therefore, they cannot be applied to draw precise conclusions on PA capabilities of people.

However, this study is able to provide researchers and health promoters with a number of options for measuring capabilities that may be useful for the field of HEPA by adapting them accordingly.

All in all, our study shows that more research is needed to develop appropriate capability instruments for 
HEPA. First, these should focus on measuring PA and all its facets, including the individual (e. g. PA-related competence), social (e. g. social support for PA), and environmental (e. g. PA infrastructures and offers) conversion factors. Second, a future measure for capabilities should ideally be applicable to a broader range of different settings, populations, and age-groups, thus allowing for standardized and comparable assessments of PA intervention effectiveness.

As HEPA can be considered a functioning which is intended to be changed by interventions, a combination of measuring both capabilities and functionings (e. g. as done by Al-Janabi [34]) might be advisable in the field. This may help future researchers to identify effects of their interventions on both levels.

We were able to identify very context-specific measurement tools, which seems appropriate due to the context specific nature of the capability approach but is likely to impede the comparability of interventions effectiveness.

To strike a compromise between detailed but settingexclusive tools and overly generic instruments, there might be a need for a framework for conceptualizing and measuring capabilities for health including our aim of health enhancing PA across the life-course, as it was done with the ICECAP measurement tool [79]. Such a framework is currently in preparation, with the intention to define a number of principles that will ensure a greater amount of comparison between age groups and settings while still allowing for the use of adapted instruments in different contexts (Till M, Gelius P, Abu-Omar K, Abel T: Using the capability approach in health promotion projects: a framework for implementation, Under review).

Despite our best of efforts, this study has some limitations which need to be borne in mind when interpreting its results and drawing conclusions. First, due to the heterogeneity of the tools identified, comparing individual instruments with each other was difficult, and it was therefore not possible to recommend a single tool that, in general, could be considered to be particularly appropriate. For the same reason, a more systematic quality assessment of the primary studies, as required by the PRISMA checklist, was not possible. Further, as we only included studies on psychometric properties that came up in our initial systematic search but did not perform a second search for psychometric property measurements for all identified quantitative tools, the results shown in this paper may miss some studies. All in all, however, we are confident that this review provides a good initial overview in an innovative and increasingly relevant area of research. Having been conducted on a large number of databases and employing an additional hand search, it presents details on different types of instruments that may guide the selection of appropriate tools for specific purposes in future research projects.

\section{Conclusion}

This systematic review has shown that there is a large variety of measurement tools available which address different aspects of capabilities, target groups or contexts. Until now, there is no golden standard on how to measure capabilities for health and therefore also none for PA. The available tools vary substantially regarding their underlying assumptions, focus on capabilities, properties (e. g. language, number of items), development processes, measurement approaches, and addressees. Most of the quantitative tools have been empirically shown to be valid, reliable and responsive, but the methods employed for validation invite skepticism as to whether all instruments truly measure capabilities and/or do so in a meaningful way. At this point in time, it is not possible to recommend a single tool for general use, and health promoters may want to choose carefully or even consider adapting a tool to their specific needs. Our findings may help inform researchers about available measurement tools that represent different options on how to measure capabilities for health and well-being, and which can be used as references for the future development of a measurement tool for capabilities for health enhancing PA.

Our findings thus seem to echo Sen's own concerns about the empirical difficulties of operationalizing the capability approach $[1,80]$, as well as those of other researches who have demurred that the multidimensional, context-dependent, and normative nature of the approach can pose problems for operationalization [81-83].

These difficulties notwithstanding, the Capital4Health consortium, under whose auspices this review was conducted, is planning to contribute to the further development of capability measurement in health promotion and PA intervention research.

\footnotetext{
Abbreviations

ADL: Activities of daily living; ADRQL: Alzheimer's disease related Quality of life; BPRS: Brief Psychiatric Rating Scale; BPRS: Brief Psychiatric Rating Scale; C: Comparison; CQ-CMH: Capability Questionnaire for Community Mental Health; CTM-3: 3-Item Care Transition Measure; D: Development; DASSD: Depression Anxiety Stress Scales; EFA: Exploratory factor analysis; FG: Focus group; GAF: Global Assessment of Functioning; HEPA: Health enhancing Physical activity; ICC: Intra-class correlation coefficient; ICECAPA: ICEpop CAPability measure for adults; ICECAP-FC: ICEpop CAPability and Functioning measure; ICECAP-O: ICEpop CAPability measure for older people; ICECAP-SCM: ICEpop CAPability measure for supportive care; K6: Kessler Psychological Distress Scale; M: Mean; M: Measurement; OCAP: Oxford Capability Questionnaire; OLS: Ordinary least square; OXCAP-MH: Oxford Capability Questionnaire for Mental Health; PA: Physical Activity; PHQ8: Patient Health Questionnaire depression scale; RAS: Recovery Assessment Scale; RT: Researcher Team; SEM: Structural equation modeling; SIX: Objective Social Outcomes Index; V: Validation; WB: Well-Being
} 


\section{Acknowledgements}

We thank Leonie Birkholz for her support during the literature search.

\section{Authors' contributions}

Conceptualization, MT., KAO., and PG.; methodology, MT., KAO, PG, AKR.; Screening, MT, SF; supervision, PG, KAO; full-text analysis, MT; writing-original draft preparation MT, PG; All authors have read and agreed to the published version of the manuscript.

\section{Funding}

The research network Capital4Health [grant number: 01EL1421A-F] and the associated subproject CAPCOM [grant number: 01EL1421A] are funded by the German Federal Ministry for Education and Research (BMBF). Open Access funding enabled and organized by Projekt DEAL.

\section{Availability of data and materials}

All data used to derive the study findings are included in this published article.

\section{Ethics approval and consent to participate}

Not applicable.

\section{Consent for publication}

Not applicable.

\section{Competing interests}

The authors declare that they have no competing interests.

\section{Received: 16 July 2020 Accepted: 29 December 2020}

\section{Published online: 15 February 2021}

\section{References}

1. Sen A. Commodities and capabilities: North-Holland; 1985.

2. Ruger JP. Health capability: conceptualization and operationalization. Am J Public Health. 2010;100(1):41-9.

3. Sen A. Capability and well-being. In: the quality of life. Edn. Edited by Nussbaum, Sen. Oxford: Clarendon Press; 1993.

4. Robeyns I. The capability approach in practice*. J Polit Philos. 2006;14(3): 351-76.

5. Abel T, Frohlich KL. Capitals and capabilities: linking structure and agency to reduce health inequalities. Social Sci Med(1982). 2012;74(2):236-44.

6. Frahsa A, Abel T, Gelius P, Rütten A. The capability approach as a bridging framework across health promotion settings: theoretical and empirical considerations. Health Promot Int. 2020:daaa076. https://doi.org/10.1093/ heapro/daaa076

7. Helter TM, Coast J, Laszewska A, Stamm T, Simon J. Capability instruments in economic evaluations of health-related interventions: a comparative review of the literature. Qual Life Res. 2020;29(6):1433-64.

8. Alkire S, Qizilbash M, Comim F, Comim F, Qizilbash M, Alkire S. Introduction. In: The Capability Approach edn; 2008. p. 1-25.

9. Caspersen CJ, Powell KE, Christenson GM. Physical activity, exercise, and physical fitness: definitions and distinctions for health-related research. Public Health Rep. 1985;100(2):126-31.

10. Warburton DE, Nicol CW, Bredin SS. Health benefits of physical activity: the evidence. CMAJ. 2006;174(6):801-9.

11. Reiner M, Niermann C, Jekauc D, Woll A. Long-term health benefits of physical activity--a systematic review of longitudinal studies. BMC Public Health. 2013;13:813.

12. World Health Organization. Regional Office for E: Steps to health : a European framework to promote physical activity for health. Copenhagen: WHO Regional Office for Europe; 2007.

13. Brownson RC, Seiler R, Eyler AA. Measuring the impact of public health policy. Prev Chronic Dis. 2010;7(4):A77.

14. McKinlay JB. Paradigmatic obstacles to improving the health of populations-implications for health policy. Salud Publica de Mexico. 1998;40(4):369-79.

15. Whitehead M. Physical literacy: throughout the Lifecourse: Taylor \& Francis; 2010

16. Antonovsky A. Unraveling the mystery of health how people manage stress and stay well; 1987.

17. Moher D, Liberati A, Tetzlaff J, Altman DG, Group P. Preferred reporting items for systematic reviews and meta-analyses: the PRISMA statement. PLoS Med. 2009;6(7):e1000097.
18. McHugh ML. The chi-square test of independence. Biochemia medica. 2013; 23(2):143-9.

19. Gliem JA, Gliem RR. Calculating, interpreting, and reporting Cronbach's alpha reliability coefficient for Likert-type scales. In: 2003: Midwest Researchto-Practice Conference in Adult, Continuing, and Community; 2003.

20. Landis JR, Koch GG. The measurement of observer agreement for categorical data. Biometrics. 1977;33(1):159-74.

21. Cicchetti DV. Guidelines, criteria, and rules of thumb for evaluating normed and standardized assessment instruments in psychology. Psychol Assess. 1994;6(4):284-90.

22. McHugh ML. Interrater reliability: the kappa statistic. Biochemia Medica. 2012;22(3):276-82.

23. Weaver RR, Lemonde M, Payman N, Goodman WM. Health capabilities and diabetes self-management: the impact of economic, social, and cultural resources. Soc Sci Med. 2014:102:58-68.

24. Ndomoto L, Hibble A, Obuzor G, Nthusi N, Quine A, Chahal P, Barasa SO, Nyanja N, Tulinius C. Understanding the fundamental elements of global health: Using the sen capability approach as the theoretical framework for a health needs assessment in deprived communities. Education for health (Abingdon, England). 2018;31(1):43-7.

25. Sauter A, Curbach J, Rueter J, Lindacher V, Loss J. German senior citizens' capabilities for physical activity: a qualitative study. Health Promot Int. 2019; 34(6):1117-29.

26. Chakraborty B, Yousefzadeh S, Darak S. Haisma H: "we struggle with the earth everyday": parents' perspectives on the capabilities for healthy child growth in haor region of Bangladesh. BMC Public Health. 2020;20(1):140.

27. Petros R, Solomon P, Linz SJ, DeCesaris M, Hanrahan NP. Capabilities approach: contextualizing participants' perspectives on systems barriers to recovery. Int J Ment Health. 2016:45(4):262-78.

28. Bucki B, Spitz E, Etienne AM, Le Bihan E, Baumann M. Health capability of family caregivers: how different factors interrelate and their respective contributions using a Bayesian approach. BMC Public Health. 2016;16:364.

29. Shahtaheri RS, Nikfar S, Sari AA, Yekani Nejad MS. Cross-cultural adaptation and psychometric analysis of the Persian version of the ICEpop CAPability measure for adults capability measure in the Iranian general population. Value Health Reg Issues. 2020;21:188-93.

30. Simon J, Łaszewska A, Leutner E, Spiel G, Churchman D, Mayer S. Cultural and linguistic transferability of the multi-dimensional OxCAP-MH capability instrument for outcome measurement in mental health: the German language version. BMC Psychiatry. 2018;18:173.

31. Coast J, Flynn TN, Natarajan L, Sproston K, Lewis J, Louviere JJ, Peters TJ. Valuing the ICECAP capability index for older people. Soc Sci Med (1982). 2008;67(5):874-82

32. Al-Janabi H, Flynn TN, Coast J. Development of a self-report measure of capability wellbeing for adults: the ICECAP-A. Qual Life Res. 2012;21(1):167-76.

33. Sutton EJ, Coast J. Development of a supportive care measure for economic evaluation of end-of-life care using qualitative methods. Palliat Med. 2014; 28(2):151-7

34. Al-Janabi H. Do capability and functioning differ? A study of UK survey responses. Health Econ. 2018;27(3):465-79.

35. Anand P, Hunter G, Carter I, Dowding K, Guala F, Van Hees M. The development of capability indicators. J Human Development Capabilities. 2009:10(1):125-52.

36. Simon J, Anand P, Gray A, Rugkåsa J, Yeeles K, Burns T. Operationalising the capability approach for outcome measurement in mental health research. Soc Sci Med. 2013;98:187.

37. Sacchetto B, Aguiar R, Vargas-Moniz MJ, Jorge-Monteiro MF, Neves MJ, Cruz MA, Coimbra JA, Ornelas J. The capabilities questionnaire for the community mental health context (CQ-CMH): a measure inspired by the capabilities approach and constructed through consumer-researcher collaboration. Psychiatric Rehab J. 2016;39(1):55-61.

38. Sacchetto B, Ornelas J, Calheiros MM, Shinn M. Adaptation of Nussbaum's capabilities framework to community mental health: a consumer-based capabilities measure. Am J Community Psychol. 2018:61(1/2):32-46.

39. Kinghorn P, Robinson A, Smith RD. Developing a capability-based questionnaire for assessing well-being in patients with chronic pain. Soc Indic Res. 2015;120(3):897-916.

40. Ferrer RL, Cruz I, Burge S, Bayles B, Castilla MI. Measuring capability for healthy diet and physical activity. Ann Fam Med. 2014;12(1):46-56.

41. Anand P, Hunter G, Smith R. Capabilities and well-being: evidence based on the Sen-Nussbaum approach to welfare. Soc Indic Res. 2005;74(1):9-55. 
42. Lorgelly PK, Lorimer K, Fenwick EAL, Briggs AH, Anand P. Operationalising the capability approach as an outcome measure in public health: the development of the OCAP-18. Soc Sci Med. 2015;142:68.

43. Holst-Kristensen AW, Fonager K, Pedersen KM. Test-retest reliability of ICECAP-A in the adult Danish population. Qual Life Res. 2020;29(2):547-57.

44. Abu-Zaineh M, Woode ME. Investigating the dimensions of youth wellbeing: an exploratory structural equation Modelling approach applied to Palestine. Child Indic Res. 2018;11(1):57-78.

45. Douptcheva N, Hill AG. Functionings and capabilities as tools for explaining differences in self- assessed health: the case of women's health in Accra Ghana. African population studies / Etude de la population Africaine. 2014; 28(2):744-57.

46. Tellez J, Krishnakumar J, Bungener M, Le Galès C. Capability deprivation of people with Alzheimer's disease: an empirical analysis using a national survey. Soc Sci Med. 2016;151:56.

47. Zwierzchowski J, Panek T. Measurement of subjective well-being under capability approach in Poland. Polish Sociol Rev. 2020;210(2):157-78.

48. Grewal I, Lewis J, Flynn T, Brown J, Bond J, Coast J. Developing attributes for a generic quality of life measure for older people: preferences or capabilities? Soc Sci Med (1982). 2006;62(8):1891-901.

49. Coast J, Peters TJ, Natarajan L, Sproston K, Flynn T. An assessment of the construct validity of the descriptive system for the ICECAP capability measure for older people. Qual Life Res. 2008;17(7):967-76.

50. Flynn TN, Chan P, Coast J, Peters TJ. Assessing quality of life among British older people using the ICEPOP CAPability (ICECAP-O) measure. Appl Health Econ Health Policy. 2011;9(5):317-29.

51. Tang C, Xiong Y, Wu H, Xu J. Adaptation and assessments of the Chinese version of the ICECAP-A measurement. Health Qual Life Outcomes. 2018; 16(1):45.

52. Couzner $L$, Ratcliffe J, Crotty M. The relationship between quality of life, health and care transition: an empirical comparison in an older post-acute population. Health Qual Life Outcomes. 2012;10:69.

53. Makai P, Brouwer WBF, Koopmanschap MA, Nieboer AP. Capabilities and quality of life in Dutch psycho-geriatric nursing homes: an exploratory study using a proxy version of the ICECAP-O. Qual Life Res. 2012;21(5):801-12.

54. Davis JC, Best JR, Dian L, Khan KM, Hsu CL, Chan W, Cheung W, LiuAmbrose T. Are the EQ-5D-3L and the ICECAP-O responsive among older adults with impaired mobility? Evidence from the Vancouver falls prevention cohort study. Qual Life Res. 2017;26(3):737-47.

55. Makai $P$, Koopmanschap MA, Brouwer WB, Nieboer AA. A validation of the ICECAP-O in a population of post-hospitalized older people in the Netherlands. Health Qual Life Outcomes. 2013;11:57.

56. Horwood J, Sutton E, Coast J. Evaluating the face validity of the ICECAP-O capabilities measure: A 'think aloud' study with hip and knee arthroplasty patients. Appl Res Qual Life. 2014;9(3):667-82.

57. Hörder H, Gustafsson S, Rydberg T, Skoog I, Waern M. A Cross-Cultural Adaptation of the ICECAP-O: Test-Retest Reliability and Item Relevance in Swedish 70-Year-Olds. Societies. 2016;6(4):30-n/a.

58. Sarabia-Cobo CM, Paras-Bravo P, Amo-Setien FJ, Alconero-Camarero AR, Saenz-Jalon M, Torres-Manrique B, Sarabia-Lavin R, Fernandez-Rodriguez A Silio-Garcia T, Fernandez-Pena R, et al. Validation of the Spanish version of the ICECAP-O for nursing home residents with dementia. PLOS One. 2017; 12(1):e0169354.

59. Franklin M, Payne K, Elliott RA. Quantifying the relationship between capability and health in older people: Can't map, Won't map. Med Decision Mak. 2018;38(1):79-94.

60. Milte R, Crotty M, Miller MD, Whitehead C, Ratcliffe J. Quality of life in older adults following a hip fracture: an empirical comparison of the ICECAP-O and the EQ-5D-3 L instruments. Health Qual Life Outcomes. 2018;16(1):173.

61. Mitchell PM, Caskey FJ, Scott J, Sanghera S, Coast J. Response process validity of three patient reported outcome measures for people requiring kidney care: a think-aloud study using the EQ-5D-5L ICECAP-A and ICECAPO. BMJ Open. 2020;10(5):e034569.

62. Baji P, Farkas M, Dobos A, Zrubka Z, Gulacsi L, Brodszky V, Rencz F, Pentek M. Capability of well-being: validation of the Hungarian version of the ICECAP-A and ICECAP-O questionnaires and population normative data. Qual Life Res. 2020;29(10):2863-74.

63. Al-Janabi H, Keeley T, Mitchell P, Coast J. Can capabilities be self-reported? A think aloud study. Soc Sci Med (1982). 2013;87:116-22.
64. Al-Janabi H, Peters TJ, Brazier J, Bryan S, Flynn TN, Clemens S, Moody A, Coast J. An investigation of the construct validity of the ICECAP-A capability measure. Qual Life Res. 2013;22(7):1831-40.

65. Al-Janabi H, Van Exel J, Brouwer W, Trotter C, Glennie L, Hannigan L, Coast J. Measuring health spillovers for economic evaluation: a case study in meningitis. Health Econ. 2016;25(12):1529-44.

66. Keeley T, Al-Janabi H, Nicholls E, Foster NE, Jowett S, Coast J. A longitudinal assessment of the responsiveness of the ICECAP-A in a randomised controlled trial of a knee pain intervention. Qual Life Res. 2015;24(10):2319-31.

67. Goranitis I, Coast J, Al-Janabi H, Latthe P, Roberts TE. The validity and responsiveness of the ICECAP-A capability-well-being measure in women with irritative lower urinary tract symptoms. Qual Life Res. 2016;25(8):2063-75.

68. Goranitis I, Coast J, Day E, Copello A, Freemantle N, Seddon J, Bennett C, Frew E. Measuring health and broader well-being benefits in the context of opiate dependence: the psychometric performance of the ICECAP-A and the EQ-5D-5L. Value Health. 2016;19(6):820-8.

69. Mitchell PM, Al-Janabi H, Byford S, Kuyken W, Richardson J, lezzi A, Coast J. Assessing the validity of the ICECAP-A capability measure for adults with depression. BMC Psychiatry. 2017;17(1):46.

70. Linton MJ, Mitchell PM, Al-Janabi H, Schlander M, Richardson J, lezzi A, Ubels J, Coast J. Comparing the German Translation of the ICECAP-A Capability Wellbeing Measure to the Original English Version: Psychometric Properties across Healthy Samples and Seven Health Condition Groups. Appl Res Qual Life. 2020;15:651-73.

71. Mah C, Noonan VK, Bryan S, Whitehurst DGT. Empirical Validity of a Generic, Preference-Based Capability Wellbeing Instrument (ICECAP-A) in the Context of Spinal Cord Injury. Patient. 2020. https://doi.org/10.1007/s40271-020-00451-6.

72. Nussbaum M, Sen A. The Quality of Life: Clarendon Press; 1993.

73. Laszewska A, Schwab M, Leutner E, Oberrauter M, Spiel G, Simon J. Measuring broader wellbeing in mental health services: validity of the German language OxCAP-MH capability instrument. Qual Life Res. 2019; 28(8):2311-23.

74. Vergunst F, Jenkinson C, Burns T, Anand P, Gray A, Rugkåsa J, Simon J. Psychometric validation of a multi-dimensional capability instrument for outcome measurement in mental health research (OxCAP-MH). Health Qual Life Outcomes. 2017;15(1):250.

75. Davis JC, Liu-Ambrose T, Richardson CG, Bryan S. A comparison of the ICECAP-O with EQ-5D in a falls prevention clinical setting: are they complements or substitutes? Qual Life Res. 2013;22(5):969-77.

76. Nussbaum M. Women's capabilities and social justice. J Hum Dev. 2000;1 (2): 219-47.

77. Al-Janabi H, Flynn TN, Peters TJ, Bryan S, Coast J. Test-retest reliability of capability measurement in the UK general population. Health Econ. 2015; 24(5):625-30

78. Lorgelly $\mathrm{P}$, Lorimer K, Fenwick E, Briggs A. The capability approach: developing an instrument for evaluating public health interventions. Glasgow: University of Glasgow, Section of public health and health policy; 2008.

79. Coast J. Assessing capability in economic evaluation: a life course approach? Eur J Health Econ. 2019;20(6):779-84

80. Sen A. Inequality reexamined: Russell Sage Foundation; 1992.

81. Sugden R. Welfare, resources, and capabilities: a review of inequality reexamined by Amartya Sen. J Econ Lit. 1993;31(4):1947-62.

82. Ysander B. Robert Erikson: descriptions of inequality. In: quality of life. Edn. Edited by Nussbaum M, Sen a. Oxford: Clarendon Press; 1993.

83. Srinivasan TN. Human development: a new paradigm or reinvention of the wheel? Am Econ Rev. 1994;84(2):238-43.

\section{Publisher's Note}

Springer Nature remains neutral with regard to jurisdictional claims in published maps and institutional affiliations. 